\title{
Micromechanical model for prediction of the fatigue limit for unidirectional fibre composites
}

\author{
Sørensen, Bent F.; Goutianos, Stergios
}

Published in:

Mechanics of Materials

Link to article, DOI:

10.1016/j.mechmat.2019.01.023

Publication date:

2019

Document Version

Peer reviewed version

Link back to DTU Orbit

Citation (APA):

Sørensen, B. F., \& Goutianos, S. (2019). Micromechanical model for prediction of the fatigue limit for unidirectional fibre composites. Mechanics of Materials, 131, 169-187.

https://doi.org/10.1016/j.mechmat.2019.01.023

\section{General rights}

Copyright and moral rights for the publications made accessible in the public portal are retained by the authors and/or other copyright owners and it is a condition of accessing publications that users recognise and abide by the legal requirements associated with these rights.

- Users may download and print one copy of any publication from the public portal for the purpose of private study or research.

- You may not further distribute the material or use it for any profit-making activity or commercial gain

- You may freely distribute the URL identifying the publication in the public portal

If you believe that this document breaches copyright please contact us providing details, and we will remove access to the work immediately and investigate your claim. 
Accepted Manuscript

Micromechanical model for prediction of the fatigue limit for unidirectional fibre composites

Bent F. Sørensen, Stergios Goutianos

PII:

S0167-6636(18)30669-0

DOI:

https://doi.org/10.1016/j.mechmat.2019.01.023

Reference:

MECMAT 2995

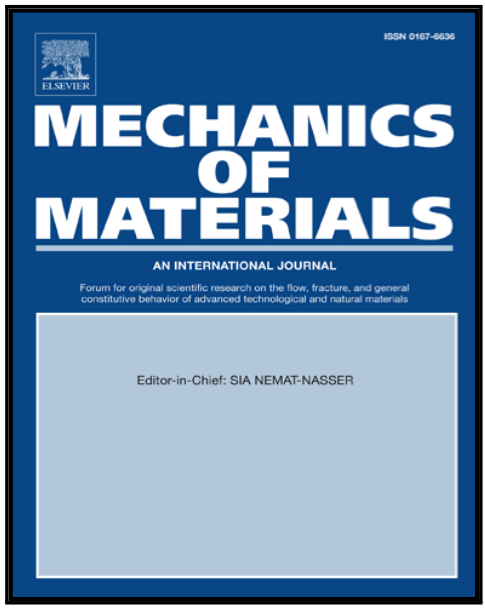

To appear in:

Mechanics of Materials

Please cite this article as: Bent F. Sørensen, Stergios Goutianos, Micromechanical model for prediction of the fatigue limit for unidirectional fibre composites, Mechanics of Materials (2019), doi: https://doi.org/10.1016/j.mechmat.2019.01.023

This is a PDF file of an unedited manuscript that has been accepted for publication. As a service to our customers we are providing this early version of the manuscript. The manuscript will undergo copyediting, typesetting, and review of the resulting proof before it is published in its final form. Please note that during the production process errors may be discovered which could affect the content, and all legal disclaimers that apply to the journal pertain. 


\section{Highlights}

- The model shows that the change in the debond crack tip stress intensity factor is zero if there is a sticking friction zone at the debond crack tip. This has very important consequences, implying that cyclic crack growth of the Paris-Erdogan type cannot occur. With sticking friction at the tip of the debond no cyclic crack growth is expected under cyclic loading. This explains the experimental observation (a) given above.

- An equation is given for the prediction of a fatigue limit expressed in terms of maximum strain as a function of basic composite and interface parameters.

- Model predictions, based on independent micromechanical experiments, show that the fatigue limit will increase for higher R-ratio. This prediction is consistent with the experimental findings.

- The model predicts that the fatigue limit will decrease with increasing fibre volume fraction. This prediction is consistent with the experimental findings. 


\title{
Micromechanical model for prediction of the fatigue limit for
}

\section{unidirectional fibre composites}

\author{
Bent F. Sørensen ${ }^{\#}$ and Stergios Goutianos \\ Section of Composites Mechanics, DTU Wind Energy, \\ The Technical University of Denmark, Ris $\varnothing$ Campus, \\ Frederiksborgvej 399, DK-4000 Roskilde, Denmark, European Union \\ \#e-mail: bsqr@dtu.dk \\ Abstract
}

\begin{abstract}
A theoretical model is developed for the prediction of a fatigue limit of unidirectional fibre composites subjected to cyclically varying tensile loads in the fibre direction. The fatigue limit is defined from the condition that failure of one fibre does not lead to progressive failure of the neighbouring fibres. The model describes the fibre/matrix interface in terms of a fracture energy and a frictional sliding shear stress. Fatigue damage in the form of cyclic debond crack growth and a decrease in the frictional sliding shear stress along the fibre matrix interface is considered. Describing the fibre strength variation in terms of a Weibull distribution, an equation for the fatigue limit is developed. The model is applied for the prediction of the fatigue limit for glass fibre composites. Effects on the fatigue limit of R-ratio, fibre volume fraction, residual stress, interfacial fracture energy as well as the interfacial frictional sliding stress and its decrease over long time forward-reverse slip are investigated. A good agreement
\end{abstract}




\section{is found between model predictions of a fatigue limit and experimental data from the literature (run-out at $10^{6}$ to $10^{10}$ cycles).}

Key words: fatigue, fibre failure, Weibull, interface, debonding, friction, residual stress

\section{Introduction}

Fibre composites based on long aligned fibres are widely used in important engineering applications where high stiffness and low mass is of importance, e.g. in aerospace, transport vehicles and wind turbine rotor blades. Most of these structures are built to be in service for long time during which they will be subjected to varying loads. For instance, a wind turbine rotor blade undergoes in the order of 100 million rotations during its 20 years' service life and gravitational and aerodynamic forces induce cyclic loads in the load-carrying composites. Material degradation under cyclic loading (fatigue) can be described in terms of the relationship between the maximum applied cyclic stress and the associated number of cycles to failure (the fatigue life), often depicted as a S-N diagram, as illustrated in Fig. 1.1. From a practical point of view, an engineering fatigue limit can be defined as run-out (surviyal) of test specimens for a relevant number of load cycles, e.g. $10^{6}$ or $10^{7}$ cycles. It is, however, an intriguing task to clarify whether or not a fatigue limit exits. Sørensen et al. (2002) suggested that the existence of a true fatigue limit should be associated with the fulfilment of two criteria: (1) The number of cycles defined as run-out should exceed the highest number of cycles that caused fatigue failure by at least a factor of ten and (2) All damage indicators, i.e., materials responses related to the state of damage, should remain unchanged during the last $90 \%$ of the number of cycles defined as run-out. Despite the importance, there are only few theoretical models that allow for the prediction of a fatigue limit for fibre composites from basic properties of fibre, matrix and the fibre/matrix interface. One model established for ceramic matrix 
composites ascribes a fatigue limit to a limiting value of the interfacial frictional shear stress (Rouby and Reynaud, 1993), assuming that the fibres retain their strength during cyclic loading. The model, however, does not include residual stress and treats the fibre/matrix interface as purely frictional (i.e., ignores fibre/matrix debonding).

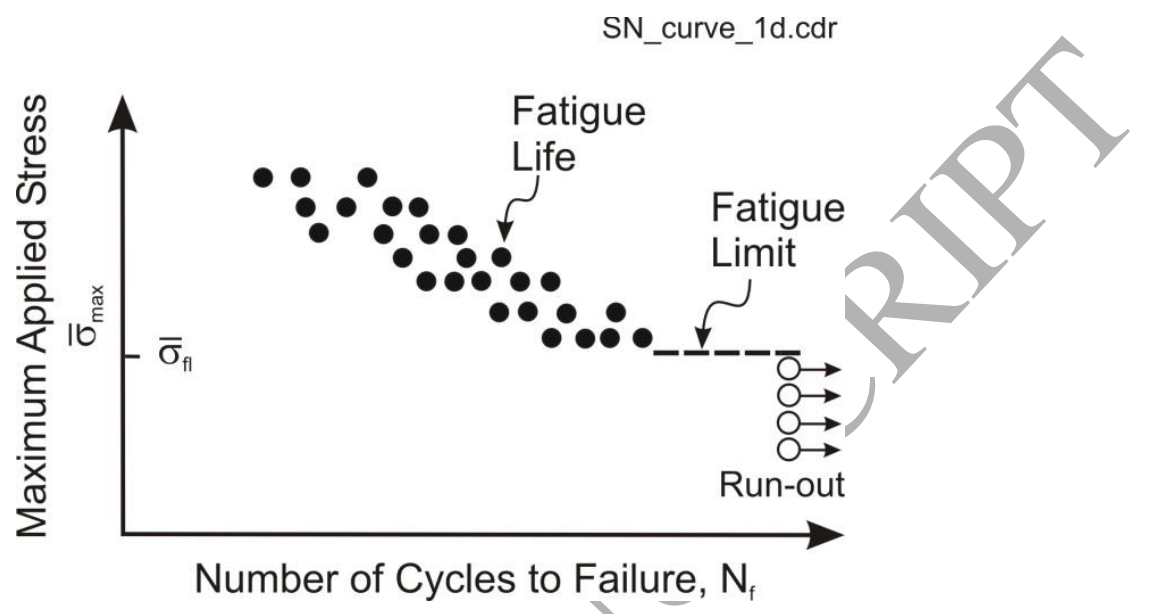

Fig. 1.1: Schematics of an S-N graph. An engineering fatigue limit can be defined based on run-out of specimens after a given number of cycles.

Another micromechanical model, representing the debond crack propagation in terms of a ParisErdogan (1963) type, was proposed by Pupurs et al. (2013). Neglecting friction along the fibre/matrix interface, the crack growth rate was predicted to decrease and approach a constant value for long crack lengths. The model of Pupurs et al. (2013) thus predicts that in the absence of interfacial friction the debond crack growth never stops under cyclic loading.

Recently, Alyes and Pimenta (2018) have presented a computational micromechanical model for the prediction of fatigue life and fatigue limit. The model describes both static and cyclic fibre/matrix crack tip debonding in terms of a cohesive law and neglects frictional sliding shear stresses along debonded interfaces. Using a Paris-Erdogan law with a threshold, the model is capable of predicting both a fatigue life and a fatigue limit. 
Other models for describing the fatigue behaviour of engineering composites are purely phenomenological in terms of macroscopic data obtained by testing of standard coupon specimens. It is useful to present macroscopic fatigue data in terms of a fatigue life diagram (Talreja, 1981), that shows the maximum strain as a function of the number of cycles to failure while distinguishing between three regions of different damage mechanisms. Strain (rather than stress) is preferred since, in a unidirectional composite, the fibre and matrix undergo identical axial straining; therefore qualitative comparisons of damage mechanisms based on applied strain are likely to be less sensitive to fibre volume fraction than comparisons based on applied stress.

There are relative few studies on fatigue of unidirectional composite materials that include data on very high number of load cycles. Typically, experiments without failure are terminated after $10^{6}$ to $10^{7}$ cycles (run-out). Mandell et al. (2003) studied high-cycle fatigue of unidirectional E-glass fibres in a polyester matrix. Smallstrands specimens that included only 45 fibres (the fibre volume fraction was 0.55 ) were tested at a loading frequency of up to $200 \mathrm{~Hz}$ and an R-ratio of 0.1 . Run-out at $10^{10}$ cycles was found for specimens tested at a maximum strain in the range of $1.2-1.5 \%$, which was somewhat higher than the range found for specimens cut from a regular laminate. For the latter run-out at $10^{8}$ occurred for a maximum strain of $0.4-0.7 \%$. This discrepancy led Mandell et al. (2003) to state that it would be wrong to use the small strand data to establish allowable strains for design. Strain values corresponding to a life of $10^{6}$ cycles $(R=0.1)$, determined experimentally for various fibre volume fraction ranging from $31 \%$ to $62 \%$, were presented by Samborsky et al. (2008). The run-out fatigue limit strain was found to decrease with increasing fibre volume fraction. In particular, the fatigue limit strain decreased sharply for fibre volume fractions in the range of 40- 
$50 \%$. Data from the OptiDat database (Nijssen, 2012) suggest that the run out at $2 \times 10^{6}$ cycles $(R=$ 0.1 ) is approximately $0.5 \%$ for unidirectional glass/epoxy composites with a fibre volume fraction of $53 \%$. None of these studies showed clear evidence of a true fatigue limit; a higher number of cycles for run-out lead to a lower fatigue limit.

Experimental investigations of R-ratio effects on possible fatigue limit are scarce. Mandell et al. (2003) found that the stress level corresponding to run-out at $10^{7}$ cycles increased significantly with increasing R-ratio for a quasi-isotropic glass/polyester laminate.

Microscopic observations of damage mechanisms in unidirectional carbon fibre composites show that fibre failure is always present (Gamstedt and Talreja, 1999). In the strain region where fatigue failure occurs, matrix cracks form perpendicular to the loading direction and bridging fibres retard the crack propagation since they create bridging tractions that tend to unload the matrix crack tip. At a lower strain region, close to the engineering fatigue limit (run-out), the dominating damage mechanism is initiation and propagation of debond cracks along the fibre/matrix interface of broken fibres. The debond cracks were found to propagate slower and slower, approaching arrest after a few million load cycles. Gamstedt and Talreja (1999) proposed that this could mark the distinction between a progressive or a stable, non-progressive fatigue mechanism: In case a debond crack continues to grow it can induce breakage of nearby fibres by increasing the stress in them. In case the debond crack stops growing (crack arrest), the damage evolution is stable and a fatigue limit may exist. A similar result (a decreasing / terminating debond crack growth rate) was also found for carbon fibre-epoxy multi-fibre model composite specimens subjected to cyclic loading between fixed maximum and minimum applied stress values (Goutianos and Peijs, 2001) and single filament 
glass fibre specimens subjected to cyclic loading between fixed maximum and minimum applied stress values, Pupurs et al. (2013).

Measurements of strains in fibres by Raman spectroscopy have shown that failure of a fibre induces additional strain in the neighbouring fibres (van den Heuvel et al., 1997) that can lead to further fibre failures. This phenomenon occurs more frequently for small fibre spacing and high fibre/matrix adhesion (interfacial fracture energy). Strain concentrations were found both at the position of the fibre break and at the end of the debonded zone (van den Heuvel et al., 1998). These findings suggest that the strain concentration depends on both fibre spacing and the interfacial fracture energy.

Building on the model by Beyerlein and Phoenix (1996), Gamstedt (2000) used a shear-lag model to predict the stress in the neighbouring fibres by a stress concentration factor, prescribing zero shear stresses along the debonded fibre/matrix interface. The stress in the neighbouring fibres was then introduced in a Weibull model (1939) and the probability of fibre failure was calculated as a function of the debond length. The probability of failure was found to increase with increasing debond length.

Using 3D finite element modelling, van den Heuvel et al. (2004) showed that a debond crack tip of a broken fibre induces a stress concentration - averaged across the fibre cross section - of about 1.05 in the neighbour fibres.

It is debatable whether glass fibres themselves develop fatigue damage. Zhou and Mallick (2004) loaded single fibres in cyclic tension and found that they experienced fatigue failure. But glass is 
known to experience subcritical crack growth in humid atmospheres (Wiederhorn, 1967). It is therefore unclear if results from fibres with uncured sizing are representative for fibres in real composites that are embedded in a matrix material that has undergone curing during the processing and thus protect the fibres better from water vapour.

The purpose of the present study is to develop a micromechanical model for the prediction of a true fatigue limit of unidirectional fibre composites along the lines of the Gamstedt-Talreja damage mechanism. We assume that the first mode of fatigue damage is isolated fibre failure with fibre/matrix debonding. We make the assumption the fibres do not develop fatigue damage. Failure of the first fibre could happen as a static overload in the very first loading.

We aim to find a condition for which the fatigue damage can be stable, viz., the debond length of the first broken fibre becomes constant and the neighbouring fibres remain unbroken. We will, based on the experimental findings by van den Heuvel et al. (1998), estimate the stress induced from the debond crack tip to it nearest neighbour fibres as the debond crack moves away from the location of the fibre break. The micromechanical model will describe the mechanics of the fibre/matrix interface in terms of (a) an interfacial fracture energy (or a critical energy release rate) and (b) a frictional sliding shear stress. The model thus allows two types of interface degradation laws: Cyclic debond crack propagation of the Paris-Erdogan type with possible crack arrest if the cyclic stress intensity factor range falls below a threshold, $\left(\Delta K_{I I}\right)_{t h}$, and a decrease in the interfacial sliding shear stress due to repeated forward and reverse sliding along the fibre/matrix interface, e.g. caused by abrasive wear of roughness asperities (Jero and Kerans, 1990; Mackin et al., 1992). The two fatigue laws are shown schematically in Fig. 1.2. As mentioned, we make the assumption that fibres do not experience fatigue degradation. Fibre failure will be assessed using a criterion for 
static tensile failure. The model will include two parts: The first part concerns the progressive development of debonding, giving mathematical relations between the applied cyclic stress and the debond length of a broken fibre. The second part sets up an equation for the fatigue limit of a unidirectional fibre composite according to the Gamstedt-Talreja mechanism.

The paper is organised as follows: First, we develop a mathematical model for the prediction of the debond length under cyclic applied stress, accounting for the possible interface fatigue degradation mechanisms described above. Having shown that the cyclic debond crack growth can be limited, the length of the debond crack is then used to set up a criterion for when a fatigue limit can exist. The model is exemplified for a glass fibre composite. Major features and limitations are discussed before the major conclusions are summarized.

\section{Problem definition}

\section{$\underline{2.1 \text { Interface laws }}$}

The mechanics of the fibre/matrix interface is described in terms of two parameters: A frictional sliding shear stress $\tau_{s}$ and an interfacial fracture energy, $\mathrm{G}^{i}$ (the critical energy release rate of the bimaterial crack tip). Following Hutchinson and Jensen (1990), who analysed fibre pull out problems, we will take the debond crack tip to be a closed Mode II crack tip. For simplicity, we assume that the second Dundurs' parameter (1969) is zero. Then the bimaterial constant is also zero and the complex bimaterial stress intensity factor decouples into classical Mode I and Mode II stress intensity factors (Rice, 1988). $\mathrm{G}^{i}$ can represent the breakage of chemical bonds (covalent bonds, ionic bonds), hydogen bonds or van der Waals, etc., and can include possible small-scale 
plastic work in the matrix at the debond crack tip (small-scale yielding). It is thus assumed that debonding can be analysed within the framework of small-scale fracture process zone.

The debond crack tip is also assumed to be able to propagate during cyclic loading. The cyclic crack growth is taken to follow a Paris-Erdogan (1963) law of the form (Kanninen and Popelar, 1985) (Fig. 1.2a)

$$
\frac{d a}{d N}=C\left(\Delta K_{I I}-\left(\Delta K_{I I}\right)_{t h}\right)^{n}
$$

where $a$ is the crack length (in the following, the crack length will be the length of the debond crack, i.e., $\left.a=\ell_{d}\right), N$ is the number of load cycles, $\Delta K_{I I}$ is the Mode II stress intensity range, defined as

$$
\Delta K_{I I}=K_{I I}^{\max }-K_{I I}^{\min }
$$

where $K_{I I}^{\max }$ and $K_{I I}^{\min }$ denote the maximum and minimum crack tip stress intensity factor values, respectively, and $\left(\Delta K_{I I}\right)_{t h}$ is a threshold value of the stress intensity factor range, implying that no cyclic crack growth takes place for smaller stress intensity factor ranges, i.e., $d a / d N=0$ for $\Delta K_{I I}<\left(\Delta K_{I I}\right)_{t h}$ (Kanninen and Popelar, 1985). In Eq. (2.1), $C$ and $n$ are material constants that are obtained by fitting the equation to experimental data. In the present study we investigate if the cyclic stress intensity range of a debond crack can fall below the threshold, $\left(\Delta K_{I I}\right)_{t h}$.

With the second Dundurs' (1969) parameter assumed to be zero, the relationship between the energy release rate and the Mode II stress intensity factor, $K_{I I}$, is given by (Rice, 1988; Hutchinson and Suo, 1992)

$$
\mathrm{G}=\frac{K_{I I}^{2}}{\bar{E}_{*}} \Rightarrow K_{I I}= \pm \sqrt{\mathrm{G} \bar{E}_{*}}
$$


where

$$
\frac{1}{\bar{E}_{*}}=\frac{1}{2}\left(\frac{1}{\bar{E}_{f}}+\frac{1}{\bar{E}_{m}}\right) .
$$

where $\bar{E}_{f}=E_{f} /\left(1-v_{f}^{2}\right)$ and $\bar{E}_{m}=E_{m} /\left(1-v_{m}^{2}\right)$ for plane strain and $\bar{E}_{f}=E_{f}$ and $\bar{E}_{m}=E_{m}$ for plane stress, where $E_{f}, E_{m}$ and $v_{f}$ and $v_{m}$ are the Young's modulus and the Poisson's ratio of the fibre and matrix (subscripts $f$ and $m$ indicate fibre and matrix), respectively. In the present paper we take $v_{f}=v_{m}$.

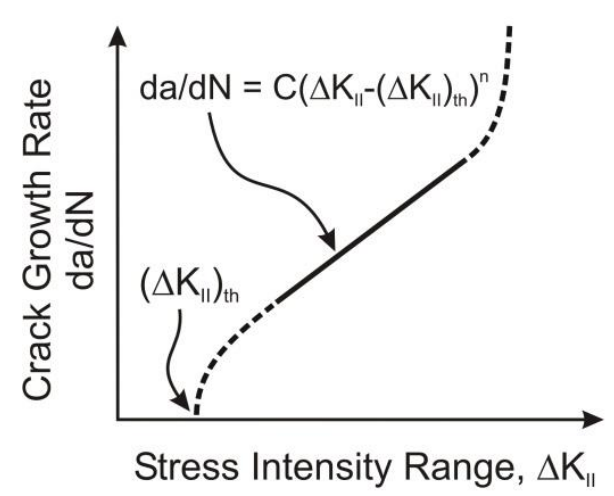

a)

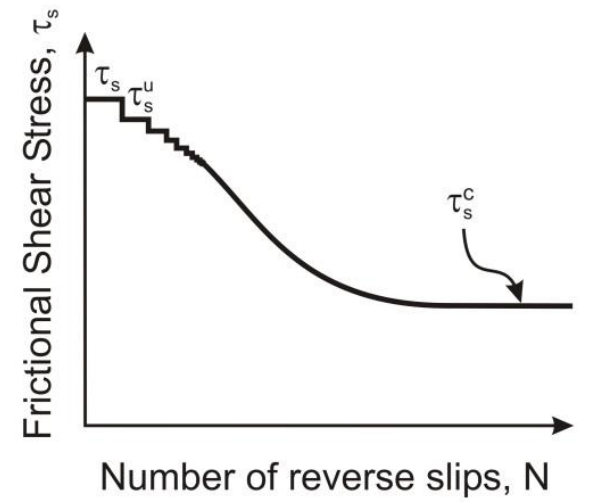

b)

Fig. 1.2: Schematics of microscale fatigue degradation laws for the fibre/matrix interface: (a) cyclic debond crack propagation described in terms of a Paris-Erdogan law and a threshold value of the stress intensity factor, and (b) a decrease in the frictional sliding shear stress.

Once debonding has occurred, the fibre can displace relative to the matrix. It is assumed that friction acts along the debonded part of the fibre/matrix interface, e.g. caused by a combination of Coulomb friction and roughness asperities along the fibre/matrix interface. We adopt the simplest possible description, describing the friction along the fibre/matrix interface in terms of a constant shear stress, $\tau_{s}$. The frictional shear stress acts opposite to the sliding direction. We assume that with increasing number of cycles of forward and reverse slip, the interfacial frictional sliding shear 
stress decreases in accordance with earlier models (Rouby and Renaud, 1993). The initial value in the first load cycle is denoted $\tau_{s}$. A lower value, $\tau_{s}^{c}$, is assumed to be attained after a high number of cycles, e.g. in the order of a million cycles where the debond crack growth has been found to cease (Gamstedt and Talreja, 1999). The assumed evolution in the frictional shear stress is sketched in Fig. 1.2b. We will not consider the details of the evolution, just the end-value, $\tau_{s}^{c}$.

\section{$\underline{2.2 \text { Unit cell model }}$}

The model that we develop is a unit cell model that consists of a single fibre having a circular cross section surrounded by a hollow matrix cylinder as shown in Fig, 2.1. The fibre is taken to have broken and debonding has taken place along a part of the fibre/matrix interface. The fibre radius is denoted $r$ and the outer radius of the matrix cylinder is $R_{m}$ so that the fibre volume fraction is

$$
V_{f}=r^{2} / R_{m}^{2}
$$

Some studies include a third (composite) cylinder outside the matrix cylinder (e.g. Pupurs and Varna (2013)) - a so called concentric cylinder assembly model - but we prefer the simpler twocylinder model in order to keep the mathematical equations tractable.

Only axial stresses (in the $z$-direction) are considered in the model. Radial and hoop stresses as well as Poisson's effects and shear deformations are ignored in this shear-lag model. These assumptions have been used in earlier analytical micromechanical models of composites under cyclic loading (McMeeking and Evans, 1990; Cho et al., 1991; Pryce and Smith, 1993; Keith and Kedward, 1995; Ahn and Curtin, 1997). 
The unit cell is essentially a model of a single filament specimen, but we will also use it to infer behaviour of a composite material with a high fibre volume fraction - in that case the model will be an approximation of a broken fibre being surrounded by intact fibres. The model is an extension of a model previously developed for analysing the single fibre fragmentation test under monotonic loading (Sørensen, 2017).

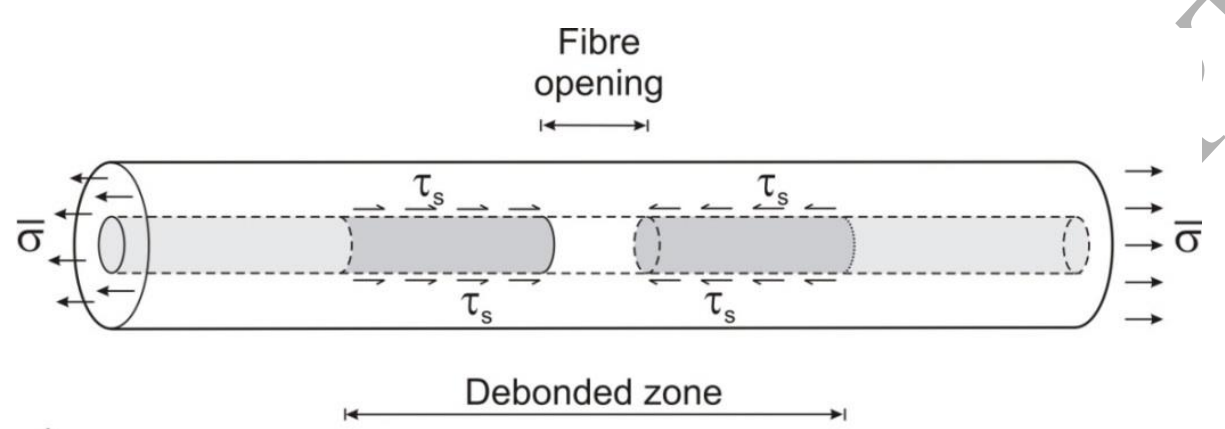

a)

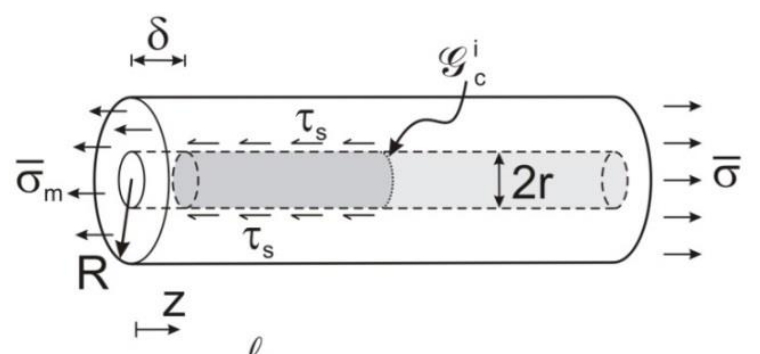

b)

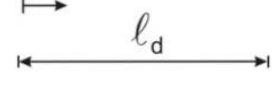

Fig. 2.1: Schematics of the symmetric problem of a single, broken fibre in a matrix cylinder (a), and (b) geometry and the basic mechanics parameters for the unit cell model (first, monotonic slip indicated).

\subsection{Synopsis of cyclic loading history}

We wish to model the behaviour of a broken fibre in a unidirectional composite subjected to cyclic loading between fixed maximum and minimum stress limits, $\bar{\sigma}_{\max }$ and $\bar{\sigma}_{\min }$. The R-ratio is

$$
R=\bar{\sigma}_{\min } / \bar{\sigma}_{\max }
$$


and the maximum cyclic strain, $\bar{\varepsilon}_{\max }=\bar{\sigma}_{\max } / E_{c}$ and minimum cyclic strain, $\bar{\varepsilon}_{\min }=\bar{\sigma}_{\min } / E_{c}$, where $E_{c}$ is the Young's modulus of the composite.

We assume that the fibre has broken at an early stage during the first loading. Then, a debond crack can propagate when the applied stress has reached a value called the debond initiation stress $\bar{\sigma}_{i}$. With increasing applied stress, the debond crack tip moves along the fibre as the applied stress $\bar{\sigma}$ increases to the maximum stress value, $\bar{\sigma}_{\max }$. The length of the debond crack will increase such that the Mode II stress intensity factor of the debond crack tip at the maximum applied stress remains at the critical value given by (2.3) with $\mathrm{G}=\mathrm{G}^{i}$ (the interfacial fracture energy):

$$
K_{I I}^{\max }= \pm \sqrt{\mathrm{G}_{c}^{i} \bar{E}_{*}}
$$

The direction of the slip at the crack tip determines whether the plus or minus sign applies. During loading the debonded part of the fibre slips in the positive $z$-direction (see Fig. 2.1) relative to the matrix. This is denoted forward slip. During this initial forward slip the interfacial shear stress has the value $\tau_{s}$ and act in the direction opposite of the forward slip direction. The debond length at a given applied stress value $\bar{\sigma}\left(>\bar{\sigma}_{i}\right)$ is called $\ell_{d}$. The fibre-end displacement $\delta$ is the difference between the displacements of the fibre-end and the matrix at the location of the fibre break, $z=0$ (see Appendix A).

Having reached the maximum applied stress, $\bar{\sigma}_{\max }$, unloading follows. The debonded interface now experiences sticking friction ("frozen" forward slip with the interfacial shear stress still being $\tau_{s}$ ), except from a zone near the location of the fibre break (position $z=0$ in Fig. 2.1), where the interface slips in the opposite direction. This is denoted reverse slip. The reverse slip length is called 
$\ell_{s}^{u}$ (superscript $u$ indicates unloading). Along the part of the debonded interface that experiences reverse slip, the interfacial shear stress acts in the opposite direction with a lower value (in accordance with Fig. 1.2b), denoted $\tau_{s}^{u}$. Unloading continues until $\bar{\sigma}$ attains the minimum applied stress value, $\bar{\sigma}_{\min }$. A schematics of the $\bar{\sigma}-\delta$ relationship is shown in Fig. 2.2 (solid curve).

SigmaDeltaCyclic_1e.cdr

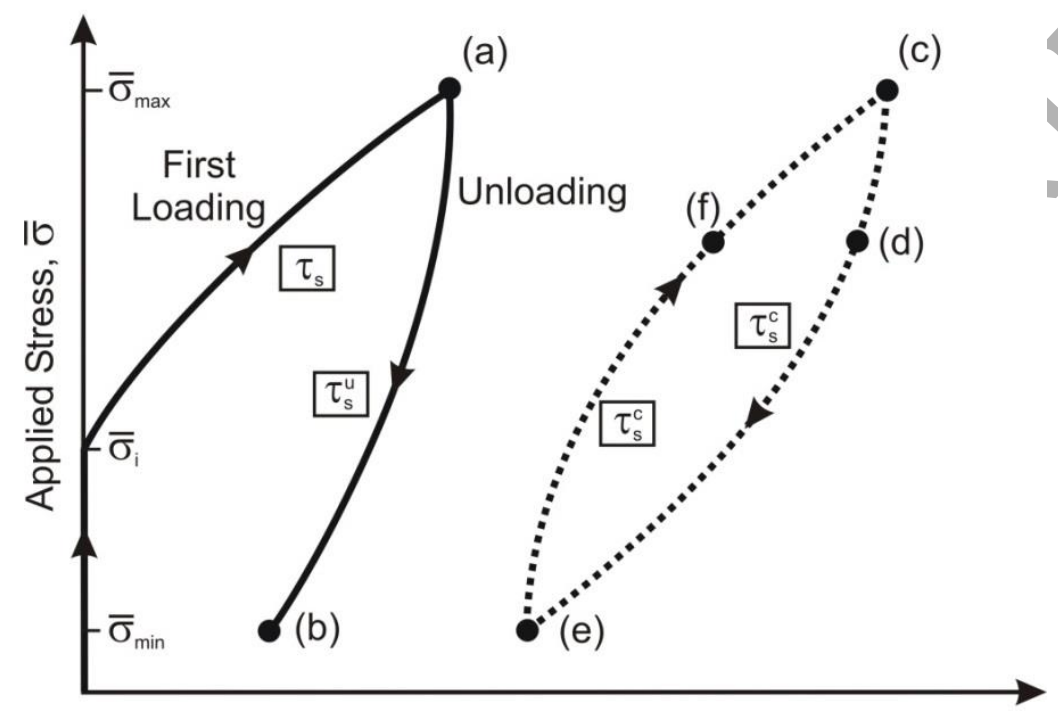

Fibre End-Displacement, $\delta$

Fig. 2.2: Schematics of parameters of applied loading history and the relationship between applied stress and the fibre-end displacement for first load cycle (solid curve), and after many load cycles (dashed). Dots and letters in parenthesis indicate situations illustrated in Fig. 2.3.

Next we jump to describe the behaviour after many load cycles. Now the interfacial shear stress has decreased to a stationary value, $\tau_{s}^{c}$, for the part of the interface where repeated forward and reverse slips occurs. During loading, the forward slip length at a given stress value, $\bar{\sigma}\left(\bar{\sigma}_{\min }<\bar{\sigma}<\bar{\sigma}_{\max }\right)$ is denoted $\ell_{s}^{l c}$. During unloading, the reverse slip length at a given applied stress is $\ell_{s}^{u c}$ and the maximum length of the reverse cyclic slip zone during unloading is $\ell_{s c}^{u c}$. The maximum forward cyclic slip zone length during loading is denoted $\ell_{s c}^{l c}$. The associated debond length after many load 
cycles is $\ell_{d}^{c}$. The dashed curve in Fig. 2.2 shows a schematics of the stress-opening displacement relationship of a broken fibre after many load cycles. Fig. 2.3 show the associated stress distribution in the fibre at maximum and minimum load of the first loading (Fig. 2.3a and Fig. 2.3.b) and at various stages during cyclic loading, after many load cycles (Fig. 2.3c - Fig. 2.3f).

\section{Part I: Model of debond and slip}

\subsection{Initial (first) loading}

The following is a summary of the major results of the model by Sørensen (2017), which describes debonding and slip under a monotonically increasing applied stress. Far ahead of the debond crack tip the stresses in the fibre and matrix are uniform, i.e. independent of $z$-position. The stress in the fibre, denoted the upstream stress, $\sigma_{f}^{+}$,

$$
\frac{\sigma_{f}^{+}}{E_{f}}=-\Delta \varepsilon^{T} \frac{\left(1-V_{f}\right) E_{m}}{E_{c}}+\frac{\bar{\sigma}}{E_{c}} \quad \text { for } z>\ell_{d},
$$

where the first term $-\Delta \varepsilon^{T}\left(1-V_{f}\right) E_{m} / E_{c}$ is the residual stress in the fibre (normalized), $\sigma_{f}^{r e s} / E_{f}$, and the second term, $\bar{\sigma} \mid E_{g}$, is the applied composite strain, $\bar{\varepsilon}_{c}$. Here $\Delta \varepsilon^{T}$ is a misfit strain, e.g. induced by thermal contraction from the processing temperature, see Sørensen (2017). Furthermore, $E_{c}$ is the Young's modulus of the composite specimen, defined as

$$
E_{c}=V_{f} E_{f}+\left(1-V_{f}\right) E_{m}
$$

The upstream stress in the matrix, $\sigma_{m}^{+}$, is given by

$$
\frac{\sigma_{m}^{+}}{E_{m}}=\Delta \varepsilon^{T} V_{f} \frac{E_{f}}{E_{c}}+\frac{\bar{\sigma}}{E_{c}} \quad \text { for } z>\ell_{d}
$$



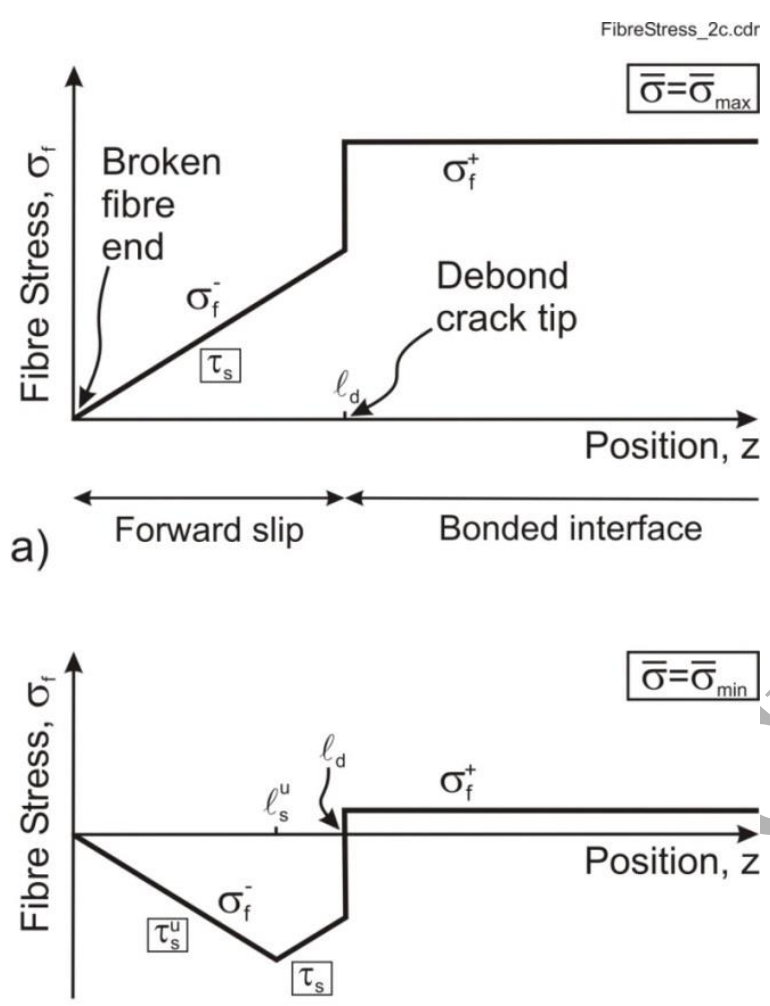

b)
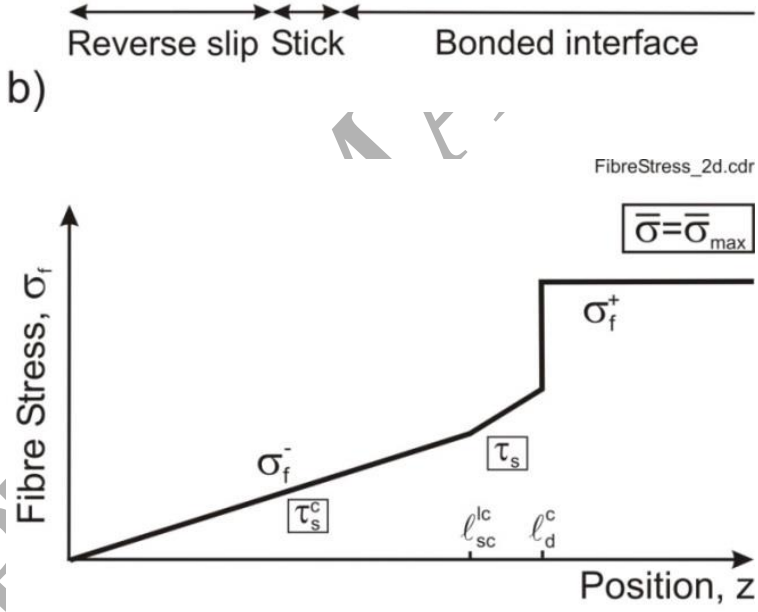

c)
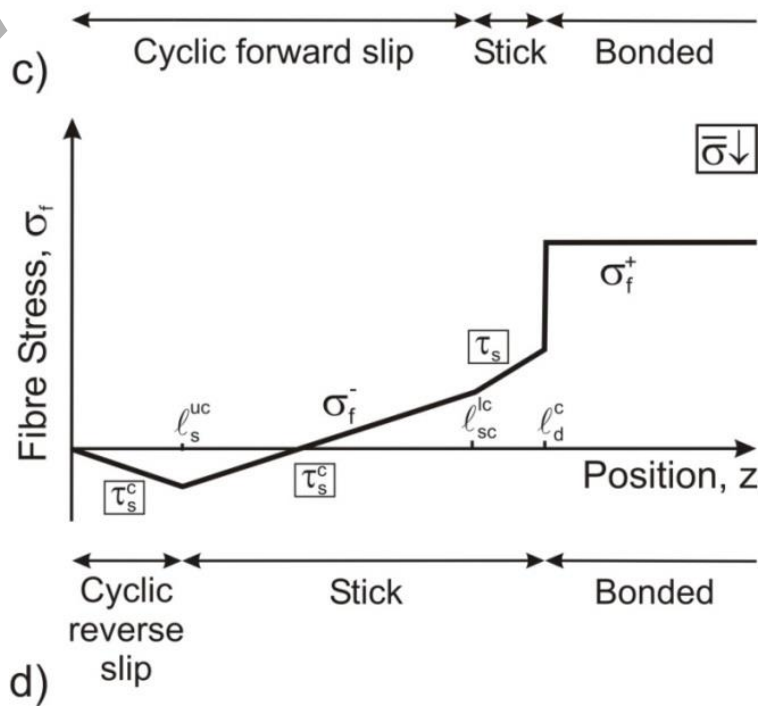
FibreStress_2d.cdr

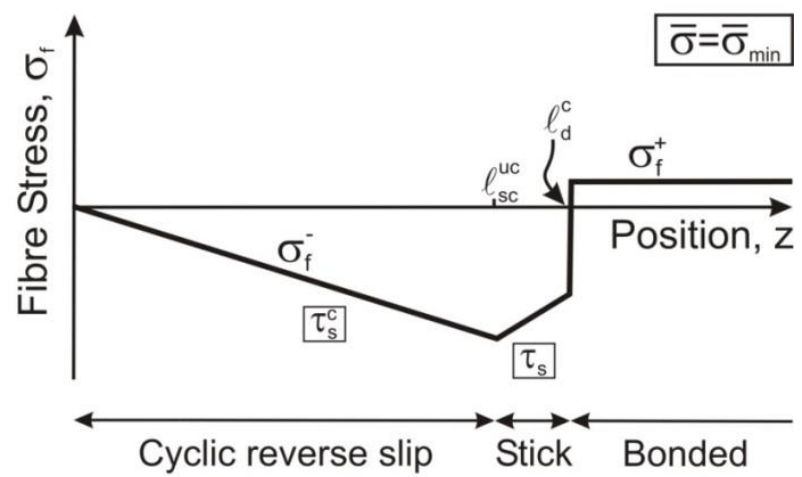

e)

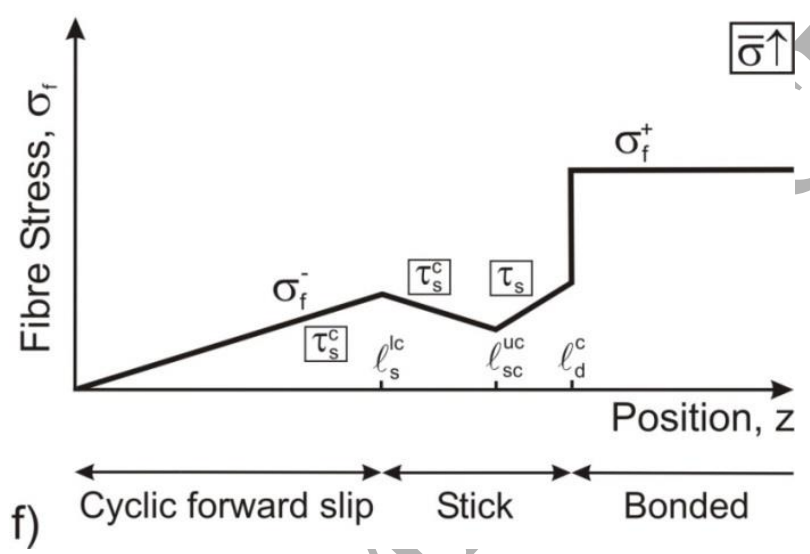

Fig. 2.3: Schematics of stress state in fibre under cyclic loading at the maximum applied stress for first loading (a), and at the minimum applied stress after first unloading (b), and after many load cycles, at the maximum applied stress (c), partially unloaded (d), at the minimum applied stress (e) and partially reloaded (f).

The first term in the right hand side of (3.3), $\Delta \varepsilon^{T} V_{f} E_{f} / E_{c}$, is the residual stress in the matrix, $\sigma_{m}^{r e s} / E_{m}$ ( normalized).

Initially, in the first loading, debond crack growth along a broken fibre will not occur until the fibre has broken and the applied stress has reached the debond initiation stress $\bar{\sigma}_{i}$, here approximated by (Sørensen, 2017) 


$$
\frac{\bar{\sigma}_{i}}{E_{c}}=\frac{\left(1-V_{f}\right) E_{m}}{E_{c}} \Delta \varepsilon^{T}+2 \sqrt{\frac{\left(1-V_{f}\right) E_{m}}{E_{c}}\left(\frac{\mathrm{G}_{c}^{i}}{E_{f} r}\right)} .
$$

For $\bar{\sigma}>\bar{\sigma}_{i}$, the debond length becomes (Sørensen, 2017)

$$
\frac{\ell_{d}}{r}=\frac{1}{2} \frac{E_{f}}{E_{c}} \frac{\left(\bar{\sigma}-\bar{\sigma}_{i}\right)}{\tau_{s}}, \quad \text { for } \bar{\sigma}>\bar{\sigma}_{i}
$$

where $\tau_{s}$ is the shear stress acting along the debonded interface in the first loading. The debond length $\ell_{d}$ is shorter than the slip length that would exist for a pure frictional interface, i.e., when the debond energy is zero (corresponding to $\bar{\sigma}_{i}=0$ in eq. (3.5)).

Within the debonded zone, $0 \leq z \leq \ell_{d}$, the stresses in the fibre and matrix, $\sigma_{f}^{-}$and $\sigma_{m}^{-}$, are given by

$$
\sigma_{f}^{-}(z)=2 \tau_{s} \frac{z}{r} \quad \text { and } \quad \sigma_{m}^{-}(z)=\frac{\bar{\sigma}}{\left(1-V_{f}\right)}-2 \frac{V_{f}}{\left(1-V_{f}\right)} \frac{z}{r} \tau_{s} \quad \text { for } 0 \leq z \leq \ell_{d} \text {. }
$$

The variation in the stress $\sigma_{f}^{-}(z)$ is sketched in Fig. 2.3a.

The displacement at the location of the fibre break $(z=0)$ was found as (Sørensen, 2017)

$$
\frac{\delta}{r}=\frac{1}{4} \frac{E_{f}}{\tau_{s}} \frac{\left(1-V_{f}\right) E_{m}}{E_{c}}\left(\frac{\bar{\sigma}}{\left(1-V_{f}\right) E_{m}}-\Delta \varepsilon^{T}\right)^{2}-\frac{\mathrm{G}^{i}}{\tau_{s} r} .
$$

The maximum debond length during the first loading is obtained by setting $\bar{\sigma}$ equal to $\bar{\sigma}_{\max }$ in eq. (3.5):

$$
\frac{\ell_{d}}{r}=\frac{1}{2} \frac{E_{f}}{E_{c}} \frac{\left(\bar{\sigma}_{\max }-\bar{\sigma}_{i}\right)}{\tau_{s}}
$$




\subsection{Stresses during first unloading $\left(\bar{\sigma}<\bar{\sigma}_{\max }\right)$}

We now consider the first unloading, i.e. when the applied stress $\bar{\sigma}$ starts to decrease after having reached $\bar{\sigma}_{\max }$. The stresses in the uncracked part of the specimen are still described by Eq. (3.1) and (3.3). During the reverse slip zone, the stress in the fibre and matrix are given by

$$
\sigma_{f}^{-}(z)=-2 \tau_{s}^{u} \frac{z}{r} \quad \text { and } \quad \sigma_{m}^{-}(z)=\frac{\bar{\sigma}}{\left(1-V_{f}\right)}+2 \frac{V_{f}}{\left(1-V_{f}\right)} \frac{z}{r} \tau_{s}^{u} \quad \text { for } 0 \leq z \leq \ell_{s}^{u},
$$

where $\tau_{s}^{u}$ is the frictional shear stress acting along the interface experiencing reverse slip during unloading. $\tau_{s}^{u}$ is expected to be smaller than $\tau_{s}$ in accordance with Fig. 1.2b. Note that in comparison with (3.6), the signs ahead of terms involving $\tau_{s}^{u}$ are changed. This is because the slip under unloading occurs in the opposite direction to that of the first loading.

For $\ell_{s}^{u} \leq z \leq \ell_{d}$, the fibre/matrix interface experiences sticking friction. Then, the stress transfer is "frozen" to be as it was for $\bar{\sigma}=\bar{\sigma}_{\text {max }}$ at the end of the first forward slip, with the interfacial shear stress being $\tau_{s}$. The composite then behaves as if was it was a "perfect" bonded composite with a composite stiffness, $E_{c}$. This implies that the strain change in this zone is simply

$$
\Delta \varepsilon_{c}=\frac{\bar{\sigma}_{\max }-\bar{\sigma}}{E_{c}} \quad \text { for } \ell_{s}^{u} \leq z \leq \ell_{d}
$$

for both the fibre and the matrix. Then, from (3.6) we get

$$
\sigma_{f}^{-}(z)=2 \tau_{s} \frac{z}{r}-\frac{E_{f}}{E_{c}}\left(\bar{\sigma}_{\max }-\bar{\sigma}\right) \quad \text { for } \ell_{s}^{u} \leq z \leq \ell_{d}
$$

and 


$$
\sigma_{m}^{-}(z)=\frac{\bar{\sigma}}{\left(1-V_{f}\right)}-2 \frac{V_{f}}{\left(1-V_{f}\right)} \frac{z}{r} \tau_{s}+\frac{V_{f}}{\left(1-V_{f}\right)} \frac{E_{m}}{E_{c}}\left(\bar{\sigma}_{\max }-\bar{\sigma}\right) \quad \text { for } \ell_{s}^{u} \leq z \leq \ell_{d} .
$$

The reverse slip length, $\ell_{s}^{u}$, is now determined by demanding stress continuity of $\sigma_{f}^{-}$at $z=\ell_{s}^{u}$, i.e. setting at $z=\ell_{s}^{u}$ in (3.9) and (3.11), setting the two equations equal to each other and solving for $\ell_{s}^{u}$ . The result is:

$$
\frac{\ell_{s}^{u}}{r}=\frac{1}{2} \frac{E_{f}}{E_{c}} \frac{\left(\bar{\sigma}_{\max }-\bar{\sigma}\right)}{\left(\tau_{s}^{u}+\tau_{s}\right)} \quad \text { for } \bar{\sigma}_{\min }<\bar{\sigma}<\bar{\sigma}_{\max }
$$

When the applied stress has decreased to the minimum cyclic stress, $\bar{\sigma}_{\min }$, the result is:

$$
\frac{\ell_{s}^{u}}{r}=\frac{1}{2} \frac{E_{f}}{E_{c}} \frac{\left(\bar{\sigma}_{\max }-\bar{\sigma}_{\min }\right)}{\left(\tau_{s}^{u}+\tau_{s}\right)}
$$

Comparing (3.14) with (3.8) shows that unless $\bar{\sigma}_{i}$ is close to $\bar{\sigma}_{\max }, \ell_{s}^{u}$ will be smaller than $\ell_{d}$. Then, the reverse slip will only occur along a part of the débonded interface.

\subsection{Calculation of energy release rate during first unloading}

We now proceed to use the potential energy loss approach of Budiansky et al. (1986) to calculate the energy release rate and thus $K_{I I}^{\text {min }}$ of the debond crack tip after unloading to $\bar{\sigma}=\bar{\sigma}_{\text {min }}$. The approach considers the potential energy change during the transition from one state (denoted State I) to another state (State II), during which both cracking and frictional sliding occur in a body with fixed surface tractions. The approach was also used in the earlier paper (Sørensen, 2017) where details of the approach were described. In the following, only the key steps are given. According to Budiansky et al. (1986), the potential energy difference between the two states is

$$
\Pi_{I}-\Pi_{I I}=\frac{1}{2} \int_{V}\left(\sigma_{I}-\sigma_{I I}\right): M\left(\sigma_{I}-\sigma_{I I}\right) d V+\xi_{F},
$$


where $\Pi_{I}$ and $\Pi_{I I}$ are the potential energy of State I and State II, respectively. Furthermore, $\sigma_{I}$ is the stress tensor associated with State I, and $\sigma_{I I}$ is the stress tensor associated with State II, $M$ indicates an elastic operator (Hooke's law) and $V$ is the volume of the body. The frictional energy dissipation, $\xi_{F}$, is defined as

$$
\xi_{F}=\tau_{s} \int_{S_{F}}\left|\Delta u_{I-I I}\right| d S
$$

where $\left|\Delta u_{I-I I}\right|$ is the magnitude of the relative frictional slip (displacement difference between fibre and matrix) between the two states - assumed to occur monotonically during the transition from State I to State II - and $S_{F}$ is the surface area over which frictional sliding occurs.

According to Budiansky et al. (1986), the decrease in the potential energy, $\Pi_{I}-\Pi_{I I}$, will be available for crack growth (fatigue crack growth) and the associated frictional energy dissipation associated with the crack extension. Denote the energy (per unit area) available for crack growth at $\bar{\sigma}=\bar{\sigma}_{\text {min }}$ by $\mathrm{G}_{\text {min }}^{i}$. Then the energy balance at $\bar{\sigma}_{\min }$ can be written as:

$$
\Pi_{I}-\Pi_{\mu}=\mathrm{G}_{\text {inin }}^{i} 2 \pi r \Delta \ell_{d}+\xi_{F} .
$$

When we have determined $G_{\text {min }}^{i}$, we can then use (2.3) and calculate $K_{I I}^{\text {min }}$, the crack tip stress intensity factor at $\bar{\sigma}_{\text {min }}$, as

$$
K_{I I}^{\min }= \pm \sqrt{\mathrm{G}_{\text {in }}^{i} \bar{E}_{*}} .
$$

We take up the analysis for $\bar{\sigma}=\bar{\sigma}_{\min }$. We aim to determine $\Pi_{I}-\Pi_{I I}$ (the left hand side of (3.17)) we use (3.15) so that we can calculate $\mathrm{G}_{\text {min }}^{i}$. For the problem at hand, State I is the situation of the debond length $\ell_{d}$ subjected to unloading with reverse sliding along the fibre/matrix interface for $0 \leq z \leq \ell_{s}^{u}$ and sticking friction for $\ell_{s}^{u} \leq z \leq \ell_{d}$. In State II, the debond crack length is assumed to 
have increased by a small distance, $\Delta \ell_{d}$, i.e. the forward slip zone now covers $\ell_{s}^{u} \leq z \leq \ell_{d}+\Delta \ell_{d}$. Following similar arguments as given by Sørensen (2017), for the transition from State I to State II, the stress state changes only for $\ell_{d}<z<\ell_{d}+\Delta \ell_{d}$. Consequently, (3.15) becomes

$$
\Pi_{I}-\Pi_{I I}=\frac{1}{2} \int_{\ell_{d}}^{\ell_{d}+\Delta \ell_{d}}\left\{\pi r^{2} \frac{\left(\sigma_{f}^{+}-\sigma_{f}^{-}(z)\right)^{2}}{E_{f}}+\pi\left(R_{m}^{2}-r^{2}\right) \frac{\left(\sigma_{m}^{+}-\sigma_{m}^{-}(z)\right)^{2}}{E_{m}}\right\} d z+\xi_{F}
$$

Inserting $\sigma_{f}^{+}$and $\sigma_{m}^{+}$from (3.1) and (3.3) and $\sigma_{f}^{-}(z)$ and $\sigma_{m}^{-}(z)$ from (3.11) and (3.12) (all with $\left.\bar{\sigma}=\bar{\sigma}_{\text {min }}\right)$ into (3.19), using (2.5), gives:

$$
\begin{aligned}
\Pi_{I}-\Pi_{I I}= & \frac{\pi}{2} r^{2}\left\{\int _ { \ell _ { d } } ^ { \ell _ { d } + \Delta \ell _ { d } } \left\{\frac{1}{E_{f}}\left[\frac{E_{f}}{E_{c}}\left(-\Delta \varepsilon^{T}\left(1-V_{f}\right) E_{m}+\bar{\sigma}_{\max }\right)-2 \tau \frac{z}{r}\right]^{2} t\right.\right. \\
& \left.\frac{\left(1-V_{f}\right)}{V_{f} E_{m}}\left[\frac{E_{m}}{E_{c}}\left(\Delta \varepsilon^{T} V_{f} E_{f}-\frac{1}{\left(1-V_{f}\right)} \frac{E_{f}}{E_{m}} \bar{\sigma}_{\max }\right)+2 \tau_{s} \frac{V_{f}}{\left(1-V_{f}\right)} \frac{z}{r}\right]^{2}\right\} d z+\xi_{F} .
\end{aligned}
$$

All the terms with $\bar{\sigma}_{\min }$ have cancelled out. Performing the integration and neglecting higher order terms of $\Delta \ell_{d}$ gives:

$$
\begin{aligned}
\Pi_{I}-\Pi_{I I}= & \frac{\pi}{2} r^{2} \frac{1}{\left(1-V_{f}\right) E_{m}}\left\{4 \frac{E_{c}}{E_{f}} \tau_{s}^{2}\left(\frac{\ell_{d}}{r}\right)^{2}+4\left(\Delta \varepsilon^{T}\left(1-V_{f}\right) E_{m}-\bar{\sigma}_{\max }\right) \tau_{s}\left(\frac{\ell_{d}}{r}\right)\right. \\
& \left.+\frac{E_{f}}{E_{c}}\left(\Delta \varepsilon^{T}\left(1-V_{f}\right) E_{m}-\bar{\sigma}_{\max }\right)^{2}\right\} \Delta \ell_{d}+\xi_{F} .
\end{aligned}
$$

Eq. (3.21) is the potential energy loss for $\bar{\sigma}=\bar{\sigma}_{\text {min }}$, which is what we seek. This equation is exactly identical to the equation for the potential energy loss of the first loading for $\bar{\sigma}=\bar{\sigma}_{\max }$ (Sørensen, 2017) that gave the maximum debond length $\ell_{d}$ at $\bar{\sigma}_{\max }$, Eq. (3.8).

In order to assess cyclic crack growth, we proceed to calculate $K_{I I}^{\min }$ from $\mathrm{G}_{\text {min }}^{i}$. Inserting the debond length given by (3.8) into (3.21) and substituting $\bar{\sigma}_{i}$ by (3.4) leads to: 


$$
\Pi_{I}-\Pi_{I I}=\mathrm{G}^{i} 2 \pi r \Delta \ell_{d}+\xi_{F} .
$$

We now insert the right hand side of (3.22) into the left hand side of (3.17). Then we note that the two terms with the frictional energy dissipation appear identically in both sides and thus cancel out. This leads to:

$$
\mathrm{G}_{\text {nin }}^{i}=\mathrm{G}^{i}
$$

Since $\mathrm{G}_{\min }^{i}$ is the energy release rate of the debond crack tip at the minimum applied stress value, $\bar{\sigma}=\bar{\sigma}_{\min }$, we can now obtain $K_{I I}^{\min }$ using (2.3) or (3.18). Next, calculating $K_{I I}^{\max }$ from $\mathrm{G}_{\max }^{i}$ of the first loading $\left(\mathrm{G}^{i}\right)$, we find by (2.2):

$$
\Delta K_{I I}=0
$$

This result is the first main result of this paper: The change in the crack tip stress intensity factor is zero if there is a sticking friction zone at the debond crack tip. This has very important consequences. Eq. (3.24) implies that $\Delta K_{I I}<\left(\Delta K_{I I}\right)_{t h}$, so that cyclic crack growth of the ParisErdogan type, equation (2.1), cannot occur. With sticking friction at the tip of the debond no cyclic crack growth is expected under cyclic loading. This result is fundamental for the fatigue limit model developed in Section 4. The result (3.24) is verified using an accurate finite element simulation in Appendix B. We will discuss the implication of the results (3.23) and (3.24) in more details in Sections 6.3 and 6.4. The finding $\Delta K_{I I}=0$ is new; earlier models of cyclic behavior of composites (McMeeking and Evans, 1990; Cho et al., 1991; Pryce and Smith, 1993; Keith and Kedward, 1995; Ahn and Curtin, 1997) did not include a debond crack tip singularity; they all treated the interface as purely frictional. Therefore, the earlier models were not capable of analyzing the debond crack tip stress intensity factor and assess cyclic debond crack growth. Furthermore, models that include fibre/matrix debonding but neglects frictional sliding shear stress along the debonded interfaces 
(Pupurs et al., 2013; Alves and Pimenta, 2018) do not capture the sticking friction at the debond crack tip and thus do not predict $\Delta K_{I I}=0$.

\section{$\underline{\text { 3.4. Reloading after many cycles }}\left(\bar{\sigma}_{\min }<\bar{\sigma}<\bar{\sigma}_{\max }\right)$}

We now consider the situation after so many load cycles that the interfacial sliding frictional shear stress has decreased to a stable value, $\tau_{s}^{c}$, that does not change henceforth, as indicated in Fig. $1 \mathrm{~b}$. During the cycles where the frictional shear stress has decreased from $\tau_{s}$ to $\tau_{s}^{c}$, the debond crack tip has advanced, to a new (stable) position at $z=\ell_{d}^{c}$, see Fig. 2.3c-f. Also, the forward and reverse slip zones have increased in length. Thus, at the end of the previous cyclic unloading, the reverse slip has extended to a position denoted $\ell_{s}^{u c}$ (superscript $u c$ indicates unloading under cyclic loading after many cycles).

As for the first loading, during the cyclic reloading, a new forward slip zone develops from the broken fibre-end $(z=0)$. The length of the new forward slipping zone is called $\ell_{s}^{l c}$ (superscripts $l c$ indicate cyclic loading). The rest of the debonded fibre/matrix interface now experience sticking friction, i.e. the interfacial shear stress is taken to be $\tau_{s}^{c}$ for $\ell_{s}^{l c}<z<\ell_{s c}^{l c}$ and $\tau_{s}$ for $\ell_{s c}^{l c}<z<\ell_{d}^{c}$.

The stresses in the fibre and matrix in the new forward slip zone $\left(0<z<\ell_{s}^{l c}\right)$ are

$$
\sigma_{f}^{-}(z)=2 \tau_{s}^{c} \frac{z}{r} \quad \sigma_{m}^{-}(z)=\frac{\bar{\sigma}}{\left(1-V_{f}\right)}-2 \frac{V_{f}}{\left(1-V_{f}\right)} \frac{z}{r} \tau_{s}^{c} \quad \text { for } 0 \leq z \leq \ell_{s}^{l c} \text {. }
$$

At a given applied stress value, $\bar{\sigma}$, the length of the new forward slip zone is found (in a similar way as we found (3.13)) to be 


$$
\frac{\ell_{s}^{c}}{r}=\frac{1}{4} \frac{E_{f}}{E_{c}} \frac{\left(\bar{\sigma}-\bar{\sigma}_{\min }\right)}{\tau_{s}^{c}}
$$

In particular, the length of the new forward slip zone at maximum applied stress is found for $\bar{\sigma}=$ $\bar{\sigma}_{\max }$ as

$$
\frac{\ell_{s c}^{c}}{r}=\frac{1}{4} \frac{E_{f}}{E_{c}} \frac{\left(\bar{\sigma}_{\max }-\bar{\sigma}_{\min }\right)}{\tau_{s}^{c}}
$$

For $\ell_{s}^{l c}<z<\ell_{s c}^{l c}$ we have

$$
\sigma_{f}^{-}(z)=E_{f} \frac{\left(\bar{\sigma}-\bar{\sigma}_{\min }\right)}{E_{c}}-2 \tau_{s}^{c} \frac{z}{r} \quad \text { for } \quad \ell_{s}^{l c}<z<\ell_{s c}^{l c}
$$

and

$$
\sigma_{m}^{-}(z)=\frac{\bar{\sigma}_{\min }}{\left(1-V_{f}\right)}+2 \frac{V_{f}}{\left(1-V_{f}\right)} \frac{z}{r} \tau_{s}^{c}+E_{m} \frac{\left(\bar{\sigma}-\overline{\sigma_{\min }}\right)}{E_{c}} \text { for } \quad \ell_{s}^{l c}<z<\ell_{s c}^{l c} .
$$

There will be sticking friction for $\ell_{s c}^{l c}<z<\ell_{d}^{c}$ with the interfacial shear stress still is taken to be $\tau_{s}$.

By continuity of stresses at $z=\ell_{s c}^{l c}$ we find the stresses in the forward sticking zone from (3.28) and (3.29):

$$
\sigma_{f}^{-}(z)=\frac{1}{2} \frac{E_{f}}{E_{c}}\left(\left(\bar{\sigma}-\bar{\sigma}_{\min }\right)-\frac{\tau_{s}}{\tau_{s}^{c}}\left(\bar{\sigma}_{\max }-\bar{\sigma}_{\min }\right)\right)+2 \tau_{s} \frac{z}{r} \tau_{s} \quad \text { for } \ell_{s c}^{l c} \leq z \leq \ell_{d}^{c}
$$

and

$$
\sigma_{m}^{-}(z)=\frac{\bar{\sigma}}{\left(1-V_{f}\right)}+\frac{1}{2} \frac{V_{f} E_{f}}{\left(1-V_{f}\right) E_{c}}\left(\frac{\tau_{s}}{\tau_{s}^{c}}\left(\bar{\sigma}_{\max }-\bar{\sigma}_{\min }\right)-\left(\bar{\sigma}-\bar{\sigma}_{\min }\right)\right)-2 \frac{V_{f}}{\left(1-V_{f}\right)} \frac{z}{r} \tau_{s} \quad \text { for } \ell_{s c}^{l c} \leq z \leq \ell_{d}^{c} \text {. }
$$

It is assumed that $\tau_{s}^{c}$ is so low that the debond length in the present situation has exceeded the initial debond length $\ell_{d}$ so that the debond crack tip has advanced to a new position denoted $\ell_{d}^{c}$. 
We again use the approach of Budiansky et al. (1986) to determine $\ell_{d}^{c}$ at the instance the applied stress is highest, i.e., $\bar{\sigma}=\bar{\sigma}_{\max }$.

We proceed to insert the stresses $\sigma_{f}^{-}(z)$ and $\sigma_{m}^{-}(z)$ from (3.30) and (3.31), both with $\bar{\sigma}=\bar{\sigma}_{\max }$, along with the upstream stresses, $\sigma_{f}^{+}$and $\sigma_{m}^{+}$, from (3.1) and (3.3) into eq. (3.19), analogous to the derivation in Section 3.3, but with $\ell_{d}$ replaced by $\ell_{d}^{c}$. Setting the criterion for crack growth as

$$
\Pi_{1}-\Pi_{2}=2 \pi r \Delta \ell_{d} \mathrm{G}^{i}+\xi_{F}
$$

leads to

$$
\frac{\ell_{d}}{r}=\frac{1}{2} \frac{E_{f}}{\tau_{s}}\left\{\frac{\bar{\sigma}_{\max }}{E_{c}}-\frac{1}{2}\left(1-\frac{\tau_{s}}{\tau_{s}^{c}}\right) \frac{\left(\bar{\sigma}_{\max }-\bar{\sigma}_{\min }\right)}{E_{c}}-\frac{\bar{\sigma}_{i}}{E_{c}}\right\}
$$

where $\bar{\sigma}_{i}$ is still given by (3.4). Using the R-ratio given by (2.6), the equation for the cyclic debond length (3.33) can be written as

$$
\frac{\ell_{d}}{r}=\frac{1}{2} \frac{E_{f}}{\tau_{s}}\left\{\frac{\bar{\varepsilon}_{\max }}{2}\left[(1+R)+(1-R) \tau_{s} / \tau_{s}^{c}\right]-\frac{\bar{\sigma}_{i}}{E_{c}}\right\} .
$$

The second major result in this paper is (3.34): An equation for the debond length under cyclic loading given in terms of maximum applied cyclic strain, $R$-ratio and interface and composite parameters. Equation (3.34) is the one from Part I that will be used in Part II where we develop an equation for the prediction of a fatigue limit. As noted in Section 3.3, it is the circumstance that a debond length will reach a limit, a limit that does not dependent on the number of applied load cycles, that enables the existence of a fatigue limit.

\section{$\underline{3.5 \text { Unloading after many load cycles }}$}


Finally, we analyse unloading after many cycles. As before, the fibre/matrix interface experiences sticking friction along the major parts of the interface in the early stages of unloading, with a new reverse slip zone initiating from the broken fibre-end. During the unloading, the interfacial sliding shear stress is assumed to take a value denoted $\tau_{s}^{c}$ along the reverse slip zone. As before, we determine the length of the reverse slip zone by requiring continuity in $\sigma_{f}^{-}$at the end of the slip zone. Within the new reverse slip zone the stresses are given by:

$$
\sigma_{f}^{-}(z)=-2 \tau_{s}^{c} \frac{z}{r} \quad \text { for } \quad 0<z<\ell_{s}^{u c}
$$

and

$$
\sigma_{m}^{-}(z)=\frac{\bar{\sigma}}{\left(1-V_{f}\right)}+2 \frac{V_{f}}{\left(1-V_{f}\right)} \tau_{s}^{c} \frac{z}{r} \text { for } 0<z<\ell_{s}^{u c}
$$

and the reverse slip zone length is found to be

$$
\frac{\ell_{s}^{c c}}{r}=\frac{1}{4} \frac{E_{f}}{E_{c}} \frac{\left(\bar{\sigma}_{\max }-\bar{\sigma}\right)}{\tau_{s}^{c}}
$$

In particular for $\bar{\sigma}=\bar{\sigma}_{\min }$ we get

$$
\frac{\ell_{s c}^{\mu c}}{r}=\frac{1}{4} \frac{E_{f}}{E_{c}} \frac{\left(\bar{\sigma}_{\max }-\bar{\sigma}_{\min }\right)}{\tau_{s}^{c}}
$$

which is the longest reverse slip length obtained during the cyclic loading. Note that the result for $\ell_{s c}^{u c}$ (3.38) is identical to $\ell_{s c}^{l c}$, given by (3.27). The maximum reverse slip length during unloading (obtained for $\bar{\sigma} \neq \bar{\sigma}_{\min }$ ) is thus identical to the maximum forward slip length during reloading (obtained for $\bar{\sigma}=\bar{\sigma}_{\max }$ ). This is consistent with expectation for a stable cyclic situation: The cyclic forward slip zone developing during loading will just be erased by the reverse slip zone during the unloading, as indicated by Fig. 2.3c-f. In contrast, for $\ell_{s c}^{l c}<z<\ell_{d}^{c}$ (equivalent to $\ell_{s c}^{u c}<z<\ell_{d}^{c}$ ) 
there will be sticking friction. The forward and reverse slip developments will thus be invariant with increasing number of load cycles providing $\tau_{s}^{c}$ remains constant.

Within the sticking zone the fibre stress is given by

$$
\sigma_{f}^{-}(z)=2 \tau_{s}^{c} \frac{z}{r}-\frac{E_{f}}{E_{c}}\left(\bar{\sigma}_{\max }-\bar{\sigma}\right) \quad \text { for } \quad \ell_{s}^{u c}<z<\ell_{s c}^{u c}
$$

and

$$
\sigma_{m}^{-}(z)=-\frac{\bar{\sigma}_{\max }}{\left(1-V_{f}\right)}-2 \frac{V_{f}}{\left(1-V_{f}\right)} \tau_{s}^{c} \frac{z}{r}-\frac{E_{f}}{E_{c}}\left(\bar{\sigma}_{\max }-\bar{\sigma}\right) \text { for } \ell_{s}^{u c}<z<\ell_{s c}^{u c} \text {. }
$$

For $\ell_{s c}^{u c}<z<\ell_{d}^{c}$, equations (3.30) and (3.31) apply for $\sigma_{f}^{-}(z)$ and $\bar{\sigma}_{m}^{-}(z)$, since sticking friction occurs during both reloading and unloading. Then, as discussed in Section 3.3, with sticking friction along the interface ahead of the crack tip, there will be no changes in the energy release rate (or stress intensity factor) of the debond crack tip. With $\Delta K_{I I}=0$ there will be no cyclic debond crack growth. We will discuss this further in Sections 6.3 and 6.4.

\subsection{Determination of $\tau_{s}^{c}$ from experiments}

We note that (3.33) has similar form as (3.8). Subtracting (3.8) from (3.33) gives

$$
\frac{\ell_{d}-\ell_{d}}{r}=\frac{1}{4} \frac{E_{f}}{\tau_{s}}\left(\frac{\tau_{s}}{\tau_{s}^{c}}-1\right) \frac{\left(\bar{\sigma}_{\max }-\bar{\sigma}_{\min }\right)}{E_{c}} .
$$

Rewriting (3.41) leads to

$$
\frac{\tau_{s}}{\tau_{s}^{c}}=1+4 \frac{\tau_{s}}{E_{f}} \frac{E_{c}}{\left(\bar{\sigma}_{\max }-\bar{\sigma}_{\min }\right)}\left(\frac{\ell_{d}-\ell_{d}}{r}\right)
$$


Eq. (3.42) provides a way to determine $\tau_{s}^{c}$ experimentally, e.g. from single filament experiments where $\ell_{d}^{c}$ should be measured after many load cycles ( 1 million, according to Gamstedt and Talreja (1999)) and $\tau_{s}$ and $\ell_{d}$ have been determined from analysis of the first loading.

\section{Part II: Model for prediction of a fatigue limit}

\subsection{Model of the Gamstedt-Talreja fatigue mechanisms}

Next we develop a micromechanical model which is based on the Gamstedt-Talreja fatigue mechanism and the finding of the previous section that $\Delta K_{I L}=0$ in the case there is a sticking friction zone at the debond crack tip. We contemplate a set of fibres. Consider the situation just after the failure of the very first fibre, i.e., just after failure of the weakest fibre in the set.

Obviously, since we consider the very first fibre failure, none of the neighbour fibres have yet failed. Now consider the situation where the applied stress level is higher than $\bar{\sigma}_{i}$. Then, the fibre may debond along a part of the fibre/matrix interface (as explained in the previous sections). As measured experimentally by van den Heuvel et al. (1998), the debond crack tip throws additional stress on the neighbouring fibres and can cause one or more of them to fail. Assuming linear-elastic fibre and matrix materials, a singular (Mode II K-field) stress field will be present around the debond crack tip. More precisely, with increasing load cycles, the debond crack can propagate along the fibre/matrix interface to the cyclic debond crack tip position, $z=\ell_{d}^{c}$, given by (3.34). As the debond crack tip propagates away from the locus of the fibre break $(z=0)$ and moves along the fibre to $z= \pm \ell_{d}^{c}$, its singular K-field passes by the neighbouring fibres correspondingly and effectively "samples" them for defects. If one of the neighbouring fibres possesses a sufficiently 
large defect it fails. The new-broken fibre will subsequently develop a debond crack, which K-field will then "samples" its neighbouring fibres. Failure of the neighbouring fibres by the K-field of the debond crack tip is thus a progressive fatigue mechanism. Conversely, if none of the neighbouring fibres fail, the situation is stable. As noted by Gamstedt and Talreja (1999), this opens the possibility of the existence of a fatigue limit.

In the following, therefore, we set up a mathematical model to assess under which conditions the debond crack length can stabilize without inducing failure in the neighbouring fibres. In Appendix C, we use a 2D plane strain model of the crack tip (shown schematically in Fig. 4.1) to estimate the maximum stress induced in the neighbouring fibres from the K-field. The result is

$$
\hat{\sigma}_{f}^{K}=\left.\sigma_{f}^{K}\right|_{\max }=0.435 \frac{E_{f}}{E_{m}} \sqrt{\frac{\mathrm{G}^{i} \bar{E}_{*}}{d^{*}}} .
$$

Note that $\hat{\sigma}_{f}^{K}$ is independent of the applied stress, $\bar{\sigma} ; \hat{\sigma}_{f}^{K}$ is thus not a stress concentration factor.

In (4.1), $d^{*}$ is the spacing between the broken fïbre and its nearest neighbour. For fibres packed in perfect hexagonal array the fibre spacing is found by elementary geometry

$$
\frac{d^{*}}{r}=\sqrt{\frac{2 \pi}{\sqrt{3} V_{f}}-2}
$$


StressinNeighbourFibre_1c.cdr

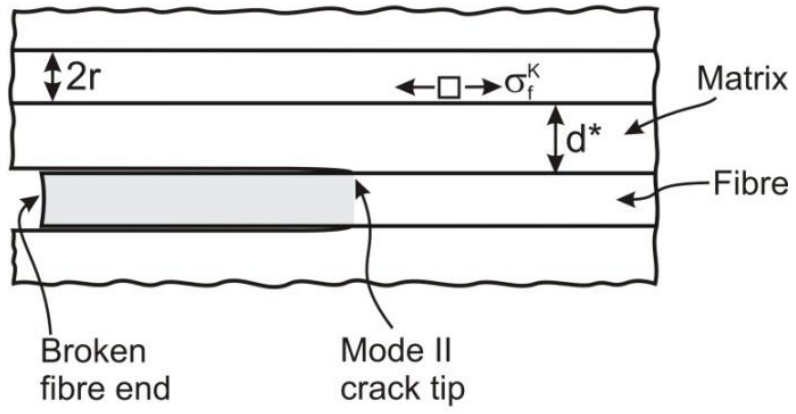

a)

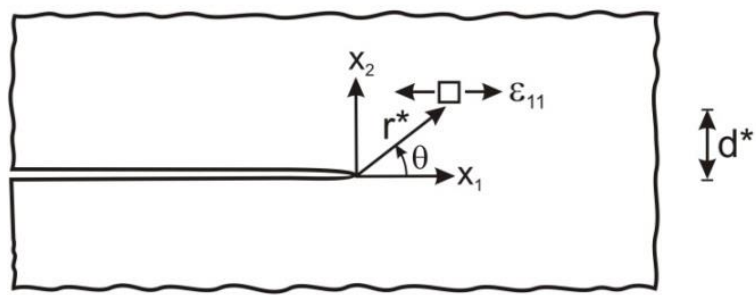

b)

Fig. 4.1: Approach for estimation of maximum stress induced in neighbouring fibres due to the debond crack tip stress field of a broken and debonded fibre (a), and the associated plane problem of a homogenous material (b).

\subsection{Weibull model for prediction of failure of neighbouring fibres}

Assuming a hexagonal fibre packing, a broken fibre will have six neighbouring fibres. Assume furthermore that the fibres fail from surface flaws. In the development of the model, we assume that the stress concentration near the debond crack tip affects only, say $1 / 6$ of the fibre surface area that is closest to the broken fibre, as indicated in Fig. 4.2. As discussed, the mechanism is only progressive if at least one of the neighbouring fibres fails. Also, we assume that the fibres fail from surface flaws. Therefore, we apply a two-parameter Weibull model (Weibull, 1939) based on fibre surface. Then, the probability of failure of a fibre with gauge length $L$ can be calculated as

$$
P_{f}(\bar{\sigma}, L)=1-\exp \left\{\frac{-1}{2 \pi r L_{0}} \int_{L}^{2 \pi} \int_{0}^{2 \pi}\left[\left(\frac{\sigma_{f}(\bar{\sigma}, \varphi)}{\sigma_{0}}\right)^{m} r d \varphi\right] d L\right\},
$$


where $\sigma_{f}(\bar{\sigma}, \varphi)$ is the stress acting around the circumference of the fibre at the applied stress value, $\bar{\sigma}$, and $L_{0}$ is the reference length of fibres used to determine the characteristic strength, $\sigma_{0}$. The Weibull modulus is denoted $m$. Furthermore, $\varphi$ is the angular coordinate (angle) around the fibre. In case the fibre stress $\sigma_{f}$ is the same all around the surface, the terms $2 \pi r$ cancel out, and (4.3) reduced to the well-known Weibull (1939) formula for constant stress.

We now wish to apply the Weibull formulation (4.3) to a model consisting of the six nearest fibres around a broken fibre, accounting for the non-uniform stress induced to the fibres by the K-field of the passing along the fibre, see Fig. 4.2 (unlike earlier models that were based on stress concentrations averaged across the fibre cross section). As shown in Fig. 4.2, we divide the arc length along each of the six surrounding fibres into two parts. A part, one sixth of the circumference of each fibre that is closest to the broken fibre, is subjected to the applied stress, the residual stress and the stress field of the K-field,

$$
\sigma_{f}^{*}\left(\bar{\varepsilon}_{\max }\right)=\sigma_{f}^{r e s}+E_{f} \bar{\varepsilon}_{\text {max }}+\hat{\sigma}_{f}^{K},
$$

where $\bar{\varepsilon}_{\max }$ is the maximum applied strain and $\hat{\sigma}_{f}^{K}$ is given by (4.1). By (3.1), Eq. (4.4) can be written as

$$
\sigma_{f}^{*}\left(\bar{\varepsilon}_{\max }\right)=\sigma_{f}^{+}\left(\bar{\varepsilon}_{\max }\right)+\hat{\sigma}_{f}^{K}
$$

The remainder of the circumference of each fibre is taken to be unaffected by the K-field of the debond crack tip of the broken fibre. For this fibre surface we take the stress to be $\sigma_{f}^{+}$. 


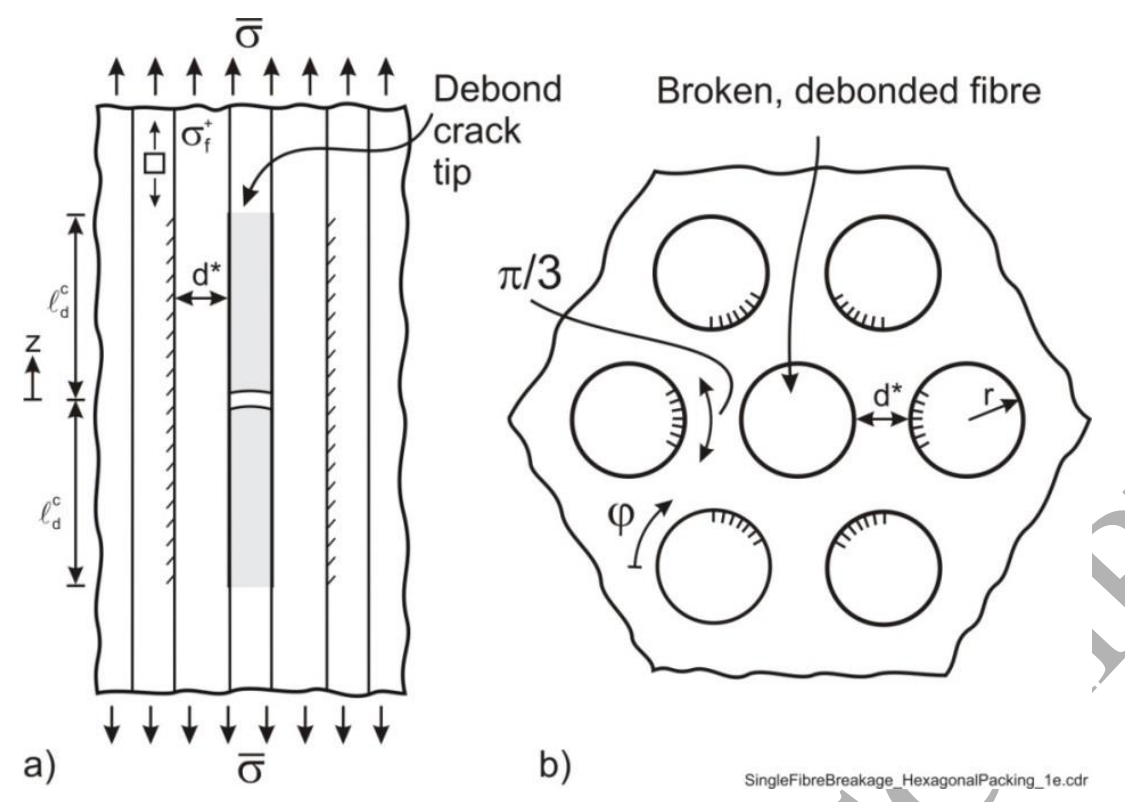

Fig. 4.2: Cross-section of six hexagonal packed fibres around a broken fibre. The fibre surface areas that have been exposed to the K-field are indicated (hatched).

Now we insert the stresses $\sigma_{f}^{+}\left(\bar{\varepsilon}_{\max }\right)$ and $\sigma_{f}^{*}\left(\bar{\varepsilon}_{\max }\right)$ from (3.1) and (4.5) into Eq. (4.3):

$$
P_{f}\left(\bar{\varepsilon}_{\max }, 2 \ell_{d}^{c}\right)=1-\exp \left\{\frac{-1}{2 \pi r L_{0}} 6 \int_{-\ell_{d}^{c}}^{\ell_{d}^{c}}\left[\int_{0}^{\pi / 3}\left(\frac{\sigma_{f}^{+}\left(\bar{\varepsilon}_{\max }\right)+\hat{\sigma}_{f}^{K}}{\sigma_{0}}\right)^{m} r d \varphi+\int_{\pi / 3}^{2 \pi}\left(\frac{\sigma_{f}^{+}\left(\bar{\varepsilon}_{\max }\right)}{\sigma_{0}}\right)^{m} r d \varphi\right] d z\right\} .
$$

In (4.6), the factor 6 is due to the presence of six fibres surrounding the broken fibre. Note also that the integration along the $z$-axis goes from $-\ell_{d}^{c}$ to $\ell_{d}^{c}$ since the fibre debonds a length $\ell_{d}^{c}$ in both directions away from the locus of the fibre fracture. The result can be written as

$$
P_{f}\left(\bar{\varepsilon}_{\text {max }}, 2 \ell_{d}^{c}\right)=1-\exp \left\{\frac{-2 \ell_{d}^{c}}{L_{0}}\left[\left(\frac{\left.\sigma_{f}^{+}\left(\bar{\varepsilon}_{\max }\right)+\hat{\sigma}_{f}^{K}\right)}{\sigma_{0}}\right)^{m}+5\left(\frac{\sigma_{f}^{+}\left(\bar{\varepsilon}_{\max }\right)}{\sigma_{0}}\right)^{m}\right]\right\} .
$$

It follows from (4.7) than an increase in $\ell_{d}^{c}$ (e.g. due to a higher value of $\bar{\varepsilon}_{\max }$ ) lead to a higher value of $P_{f}\left(\bar{\varepsilon}_{\max }, 2 \ell_{d}^{c}\right)$. 
The volume under consideration includes the surfaces of six fibres. Thus, $P_{f}$ is the probability of at least one (out of the six) fibre failing within a distance (in both directions) of $\ell_{d}^{c}$ at the maximum applied strain level, $\bar{\varepsilon}_{\max }$. Consequently, failure of one of the six surrounding fibres can be written as

$$
P_{f}\left(\bar{\varepsilon}_{\max }, 2 \ell_{d}^{c}\right)=\frac{1}{6}
$$

In the following, we denote the values of $\ell_{d}^{c}$ and $\bar{\varepsilon}_{\max }$ that simultaneously fulfil (4.7) and (4.8) by $\ell_{d}^{0}$ and $\bar{\varepsilon}_{f l}$ (subscript $f l$ indicates fatigue limit). Combining (4.7) and (4.8) gives $\ell_{d}^{0}$ as a function of $\bar{\varepsilon}_{f l}:$

$$
\ell_{d}^{0}=\frac{L_{0}}{2}\left[\left(\frac{\sigma_{f}^{+}\left(\bar{\varepsilon}_{f l}\right)+\hat{\sigma}_{f}^{K}}{\sigma_{0}}\right)^{m}+5\left(\frac{\sigma_{f}^{+}\left(\bar{\varepsilon}_{f l}\right)}{\sigma_{0}}\right)^{m}\right]^{-1} \ln \left(\frac{6}{5}\right)
$$

where the functional dependence of $\bar{\varepsilon}_{f l}$ is through $\sigma_{f}^{+}$, eq. (3.1).

The debond length, $\ell_{d}^{0}$, given by (4.9) should be understood as the debond length that - together with $\sigma_{f}\left(\bar{\varepsilon}_{f l}\right)$ - corresponds to $P_{f}=1 / 6$ and thus gives failure of one of the surrounding fibres. If the actual debond length of the cyclic problem, $\ell_{d}^{c}$, is smaller, $\ell_{d}^{c}<\ell_{d}^{0}$, then $P_{f}<1 / 6$ so that none of the neighbouring fibres fail. The situation is then stable and a fatigue limit can exist. Whether $\ell_{d}^{c}$ is smaller than $\ell_{d}^{0}$ or not can thus be taken as a criterion for the existence of a fatigue limit.

Therefore, we define the fatigue limit as the strain value for which the debond length during cyclic loading is equal (or smaller) than $\ell_{d}^{0}$ so that the debonding of the first broken fibre does not lead to breakage of a neighbouring fibre:

$$
\ell_{d}^{c}=\ell_{d}^{0}
$$


Inserting $\ell_{d}^{c}$ from (3.34) and $\ell_{d}^{0}$ from (4.9) into (4.10) leads to

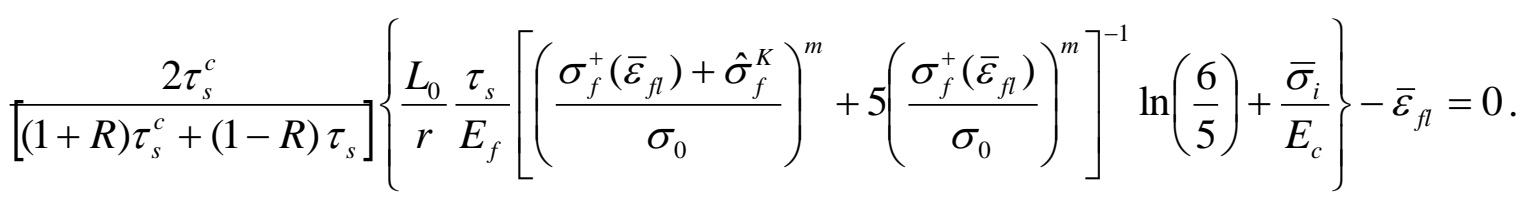

Eq. (4.11) is our third main result: The prediction of a fatigue limit expressed in terms of maximum strain as a function of basic composite and interface parameters. Eq. (4.11) includes $\bar{\varepsilon}_{f l}$ in both the two terms inside the square bracket and cannot easily be solved explicitly. But $\bar{\varepsilon}_{f l}$ can easily be determined numerically from (4.11) by the use of a Newton-Ralpson method using derivative (Press et al., 1992).

Introducing the utility parameter

$$
\mathrm{A}_{1}=\frac{2 \tau_{s}^{c}}{\left[(1+R) \tau_{s}^{c}+(1-R) \tau_{s}\right]} \quad \text { for } 0 \leq R<1 \quad \text { and } 0<\tau_{s}^{c} / \tau_{s} \leq 1
$$

(4.11) can be written as

$$
\mathrm{A}_{1}\left\{\frac{L_{0}}{r} \frac{\tau_{s}}{E_{f}}\left[\left(\frac{\sigma_{f}^{+}\left(\bar{\varepsilon}_{f l}\right)+\hat{\sigma}_{f}^{K}}{\sigma_{0}}\right)^{m}+5\left(\frac{\sigma_{f}^{+}\left(\bar{\varepsilon}_{f l}\right)}{\sigma_{0}}\right)^{m}\right]^{-1} \ln \left(\frac{6}{5}\right)+\frac{\bar{\sigma}_{i}}{E_{c}}\right\}-\bar{\varepsilon}_{f l}=0
$$

Note that the two parameters $\tau_{s}^{c}$ and $R$ appear only in the parameter $\mathrm{A}_{1}$. Thus the two parameters can (in the modelling) be taken together in $A_{1}$.

An approximate solution for high fibre volume fraction is obtained as follows. In that case, $\sigma_{f}^{+}\left(\bar{\varepsilon}_{f l}\right)$ $\ll<\hat{\sigma}_{f}^{K}$ (see Fig. 5.1) so that we can neglect the term $\sigma_{f}^{+}\left(\bar{\varepsilon}_{f l}\right)$ in (3.13). Then we find:

$$
\bar{\varepsilon}_{f l} \approx \mathrm{A}_{1}\left\{\frac{L_{0}}{r} \frac{\tau_{s}}{E_{f}}\left[\left(\frac{\sigma_{0}}{\hat{\sigma}_{f}^{K}}\right)^{m}\right] \ln \left(\frac{6}{5}\right)+\frac{\bar{\sigma}_{i}}{E_{c}}\right\}
$$


It will shown shortly that this estimate is only accurate for quite high values of $V_{f}$. Nevertheless, eq. (4.14) provides insight into the dependence of parameters. It can be seen from (4.14) that for high values of $V_{f}, \bar{\varepsilon}_{f l}$ is linearly proportional to $\mathrm{A}_{1}$, and thus it follows from (4.12) and (4.14) that $\bar{\varepsilon}_{f l}$ increases with increasing R-ratio and increasing $\tau_{s}^{c} / \tau_{s}$ ratio. Clearly, $\bar{\varepsilon}_{f l}$ also increases with increasing $\tau_{s}$ and increasing $\Delta \varepsilon^{T}$ via $\bar{\sigma}_{i}$. However, the dependency of $\mathrm{G}_{c}^{i}$ in (4.14) is not clear, since $\mathrm{G}_{i}^{i}$ affects both $\hat{\sigma}_{f}^{K}$ and $\bar{\sigma}_{i}$, see (4.1) and (3.4).

The predicted fatigue limit can also be expressed in terms of stresses, as $\bar{\sigma}_{f l}=E_{c} \bar{\varepsilon}_{f l}$ (Hooke's law), where $E_{c}$ is given by (3.2).

\section{Model results}

In this section, we apply the model for the prediction of the fatigue limit of unidirectional glassfibre composites with a polymer matrix. Representative material data, collected from various studies, are summarized in Table 1. It is acknowledged that the use of parameters from different sources induces uncertainty due to material variation from study to study.

\subsection{Effect of fibre volume fraction and mismatch strain on fibre stress}

First we estimate the residual stress in the fibre $\sigma_{f}^{\text {res }}$ and the maximum stress induced in the neighbouring fibres, $\hat{\sigma}_{f}^{K}$, from the debond crack tip as a function of the fibre volume fraction using (3.1) and (4.1) and the material data given in Table 1. Results are shown in Fig. 5.1. The residual stress is negative (compressive) and $\sigma_{f}^{\text {res }} / E_{f} \approx-0.3 \%$ for $V_{f} \approx 0$ and approaches zero for $V_{f}$ 
approaching 0.9 . In contrast, $\hat{\sigma}_{f}^{K} / E_{c}$ is positive (tensile) and takes it lowest value $(0.6 \%)$ for $V_{f} \approx 0$, and increases significantly with increasing value of $V_{f}$ reaching $2.6 \%$ for $V_{f}=0.5$. $\hat{\sigma}_{f}^{K}$ is significantly larger in magnitude than $\sigma_{f}^{\text {res }}$, in particularly for $V_{f}>0.5$.

$\underline{\text { Table } 1}$

\begin{tabular}{|c|c|c|c|c|c|c|c|}
\hline $\begin{array}{c}E_{f} \\
(\mathrm{GPa})\end{array}$ & $\begin{array}{c}E_{m} \\
(\mathrm{GPa})\end{array}$ & $\begin{array}{c}r \\
(\mu \mathrm{m})\end{array}$ & $\begin{array}{c}\mathrm{G}_{i}^{i} \\
\left(\mathrm{~J} / \mathrm{m}^{2}\right)\end{array}$ & $\begin{array}{c}\tau_{s} \\
(\mathrm{MPa})\end{array}$ & $\begin{array}{c}\Delta \varepsilon^{T} \\
(-)\end{array}$ & $\begin{array}{c}\sigma_{0} \\
(\mathrm{MPa})\end{array}$ & $\begin{array}{c}L_{0} \\
(\mathrm{~mm})\end{array}$ \\
\hline $70^{\#}$ & $4^{\#}$ & $7.5^{\#}$ & $50^{\$}$ & $33^{\#}$ & $0.346 \%{ }^{\#}$ & $4.62^{\alpha}$ & $20^{\alpha}$ \\
\hline
\end{tabular}

Properties of fibres, matrix and interface of a unidirectional glass fibre composite with polymer

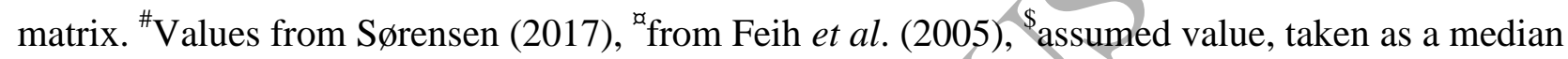
of values from Kim and Nairn (2002), Graciani et al. (2011) and Sørensen (2017).

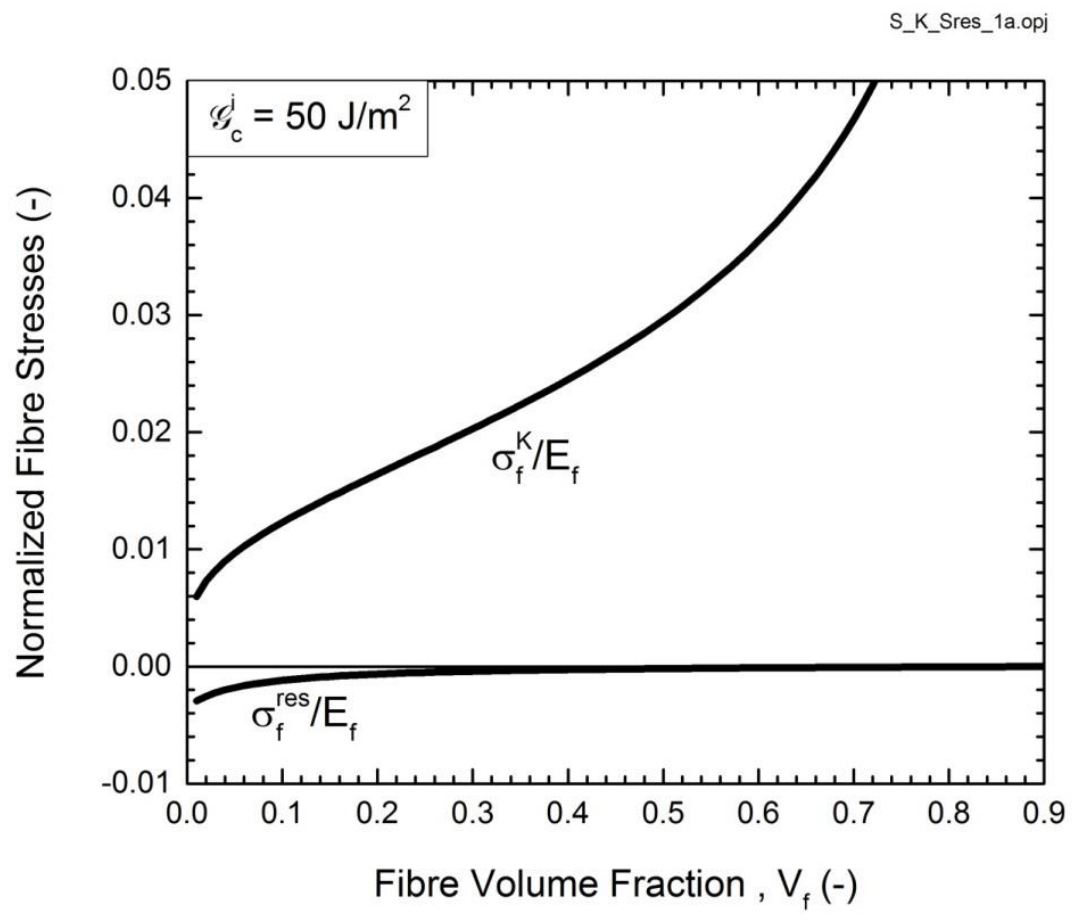

Fig. 5.1: Stresses in neighbour fibres: Comparison of residual stresses and stresses induced by the debond crack tip. 


\subsection{Effect of fibre volume fraction and mismatch strain on fatigue limit}

The predicted fatigue limit $\bar{\varepsilon}_{f l}$ for unidirectional glass fibre composites with a polymer matrix is shown as a function of the fibre volume fraction in Fig. 5.2. $\bar{\varepsilon}_{f l}$ is determined numerically from (4.13) using a Newton-Raphson method and a convergence criterion based on the difference between two successive $\bar{\varepsilon}_{f l}$ iteration values being less than $10^{-9}$. Results are shown for zero ( $\Delta \varepsilon^{T}$ $=0)$ and a realistic mismatch strain value $\left(\Delta \varepsilon^{T}=0.346 \%\right)$ and a higher mismatch strain $\left(\Delta \varepsilon^{T}=1 \%\right)$ to illustrate the effects of residual stresses. Several features in Fig. 5.2 are noteworthy. First, irrespective of the value of $\Delta \varepsilon^{T}, \bar{\varepsilon}_{f l}$ decreases with increasing $V_{f}$ in a near-linear fashion. For $V_{f}$ close to zero, $\bar{\varepsilon}_{f l}$ is above $2.5 \%$, decreasing to about $0.12 \%$ for $V_{f}=0.9$. For $V_{f} \approx 0.5$ (order of magnitude of most engineering composites), $\bar{\varepsilon}_{f l}$ is predicted to be $1.4 \%$ when $\Delta \varepsilon^{T}=0$, but for $V_{f}=$ 0.7, which can be a representative value for a fibre rich domain, $\bar{\varepsilon}_{f l}$ has decreased to only $0.66 \%$. It is also clear from Fig. 5.2 that the presence of a (negative) residual stress in the fibre increases the fatigue limit, but the effect is insignificant except for small values of $V_{f}$. Results obtained using the approximate solution (4.14) is also shown as a dashed line in Fig. 5.2. The approximate solution seems only to be accurate for quite high fibre volume fractions $\left(V_{f}>0.75\right)$ and may thus be of limited practical use.

\subsection{Effects of changes in interfacial friction and R-ratio on fatigue limit}

Next, Fig, 5.3, shows the frictional pre-factor $A_{1}$, given by (4.12), as a function of the frictional shear stress ratio $\tau_{s}^{c} / \tau_{s}$ and the R-ratio. The value of $\mathrm{A}_{1}$ lies between 0.0 and 1.0; the lowest values occur for low R-ratio values and lowest $\tau_{s}^{c} / \tau_{s}$ ratio. Generally, $\mathrm{A}_{1}$ increases with increasing 
$\tau_{s}^{c} / \tau_{s}$ and $R$, so from the point of view of $\mathrm{A}_{1}$, an increase in $\tau_{s}^{c} / \tau_{s}$ is equivalent to an increase in $R$ and vice versa.

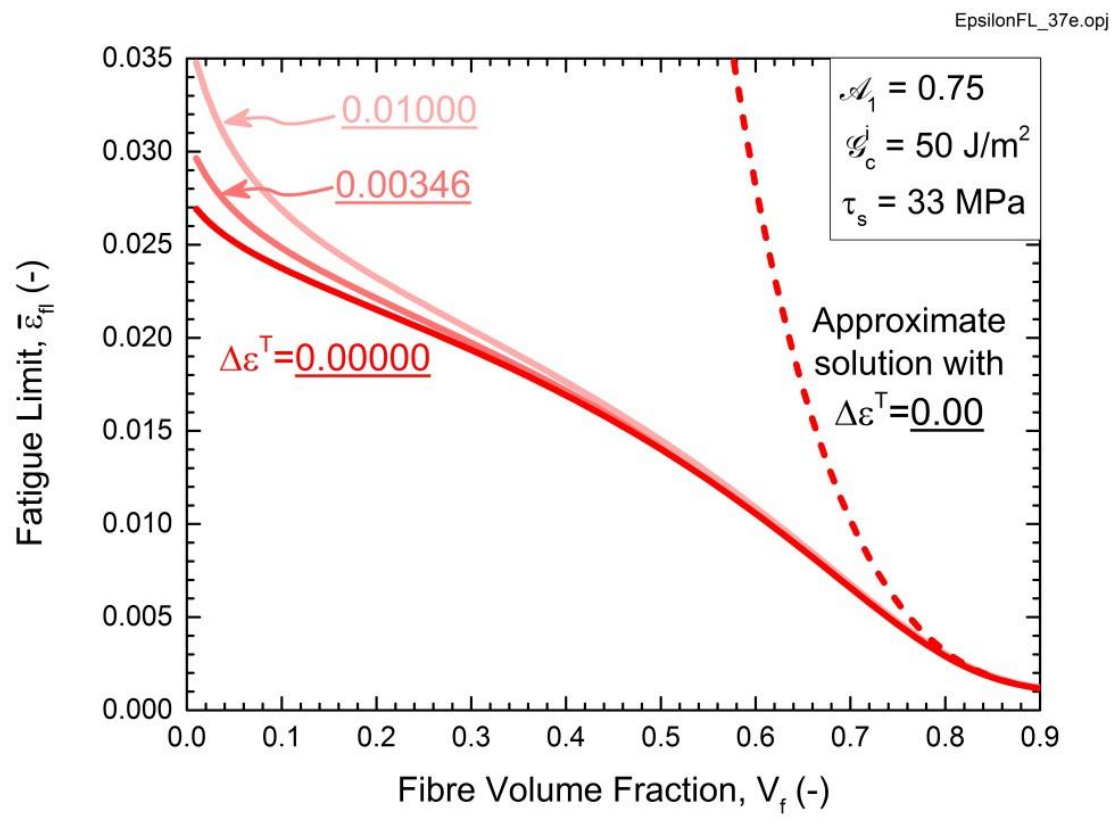

Fig. 5.2: Predicted fatigue limit as a function of fibre volume fraction for various values of the mismatch strain. Dashed curve indicates the approximate solution, eq. (4.14).

The effects of the pre-factor $A_{1}$ on the fatigue limit is shown next in Fig. 5.4. The curves show a trend similar to that of Fig. 5.2; near $V_{f}=0, \bar{\varepsilon}_{f l}$ is in the range of 2.3-3.3\%, decreasing nearlinearly with increasing for $V_{f}$ to about $0.004 \%$ to $0.16 \%$ (dependent on the pre-factor) for $V_{f}=$ 0.9. For a fixed $V_{f}$, the highest values of $\bar{\varepsilon}_{f l}$ is obtained when the pre-factor $A_{1}$ is unity and the lowest value of $\bar{\varepsilon}_{f l}$ is obtained for lowest $A_{1}$-value. The largest effect of the pre-factor is in the range of $V_{f}$ below 0.6 . For $V_{f}=0.5, \bar{\varepsilon}_{f l}$ is about $0.8 \%$ when $\mathrm{A}_{1}$ is 0.25 and $1.6 \%$ when $\mathrm{A}_{1}$ is 1.0 . 
FatigueLimit_R-ratio_2c.opj

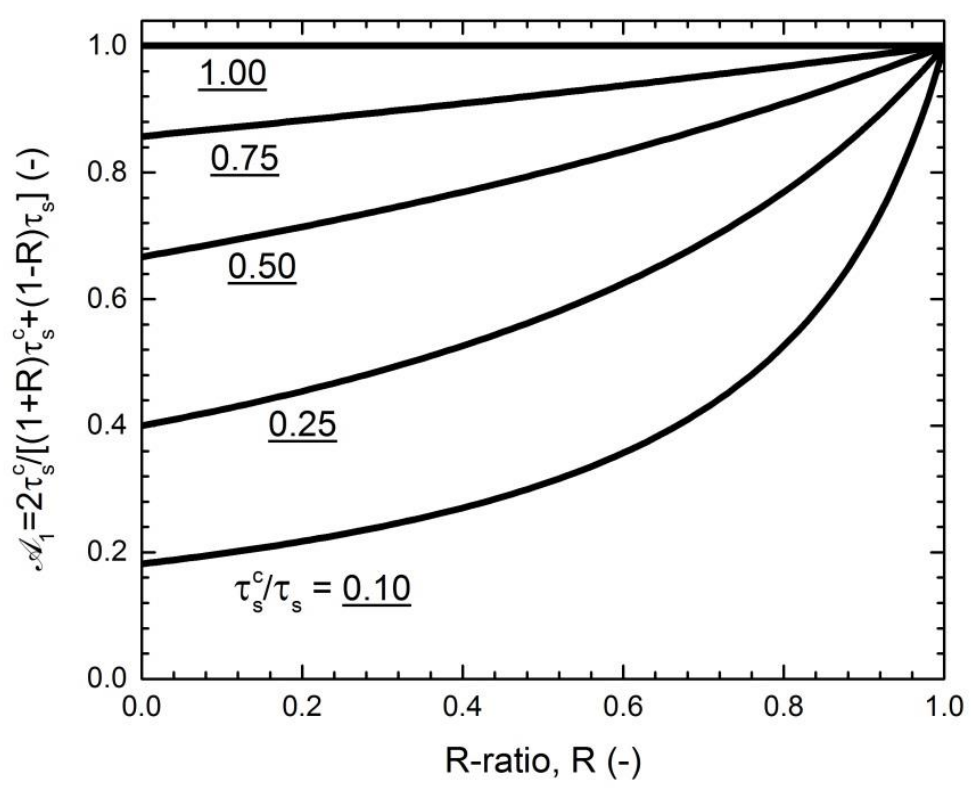

Fig. 5.3: The numerical pre-factor $A_{1}$ shown as a function of R-ratio and cyclic-to-static frictional shear stress ratio.

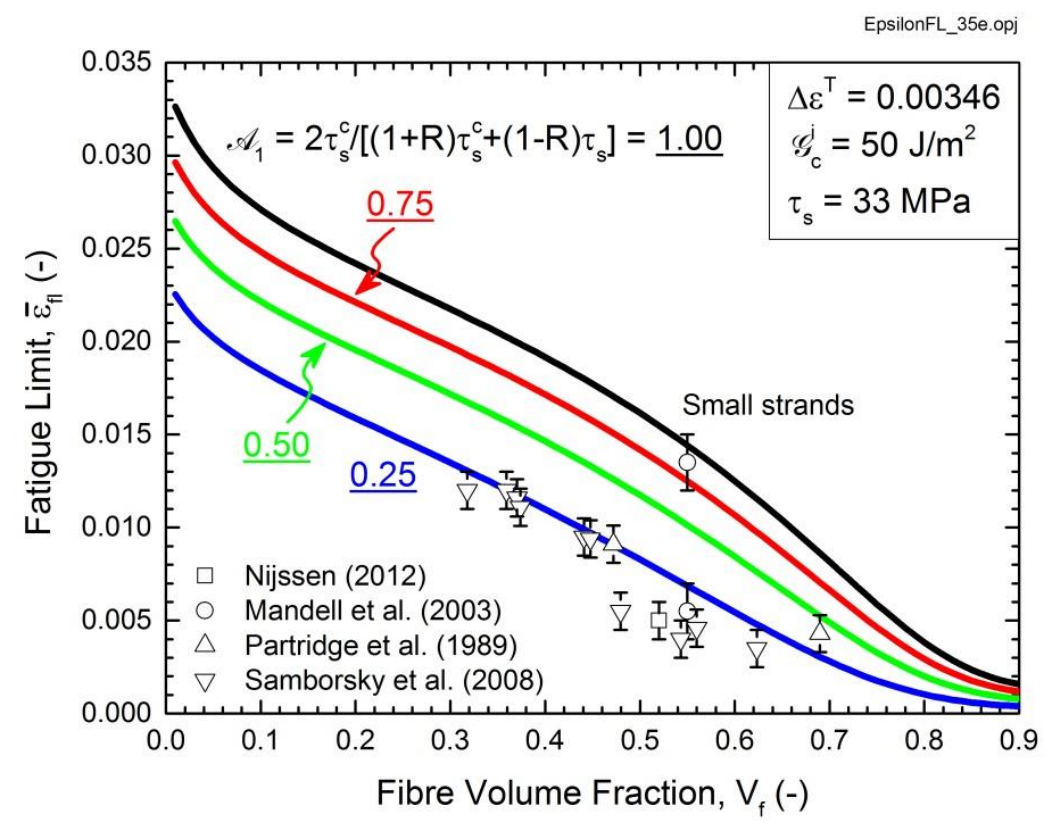


Fig. 5.4: Predicted fatigue limit expressed in terms of strain is shown as a function of fibre volume fraction for various values of the frictional pre-factor $A_{1}$. Experimental data for various glass fibre composites from several papers are superimposed as symbols.

The dependence of the fatigue limit, expressed in terms of stress, $\bar{\sigma}_{f l}$, is shown as a function of $V_{f}$ for various values of $A_{1}$ in Fig. 5.5. The predicted $\bar{\sigma}_{f l}$ increases with $V_{f}$, attains a maximum value for $V_{f}$ in the range of $0.38-0.48$ (for the present set of parameters), and decreases for higher values of $V_{f}$. Other parameters could lead to a maximum in a different range. Nevertheless, the model thus predicts that there exists an optimal value of $V_{f}$; the value of $V_{f}$ that maximizes $\bar{\sigma}_{f l}$. This prediction differs from the prediction the model of Rouby and Reynaud (1993). According to that model, the fatigue limit in terms of stress increases linearly with the fibre volume fraction.

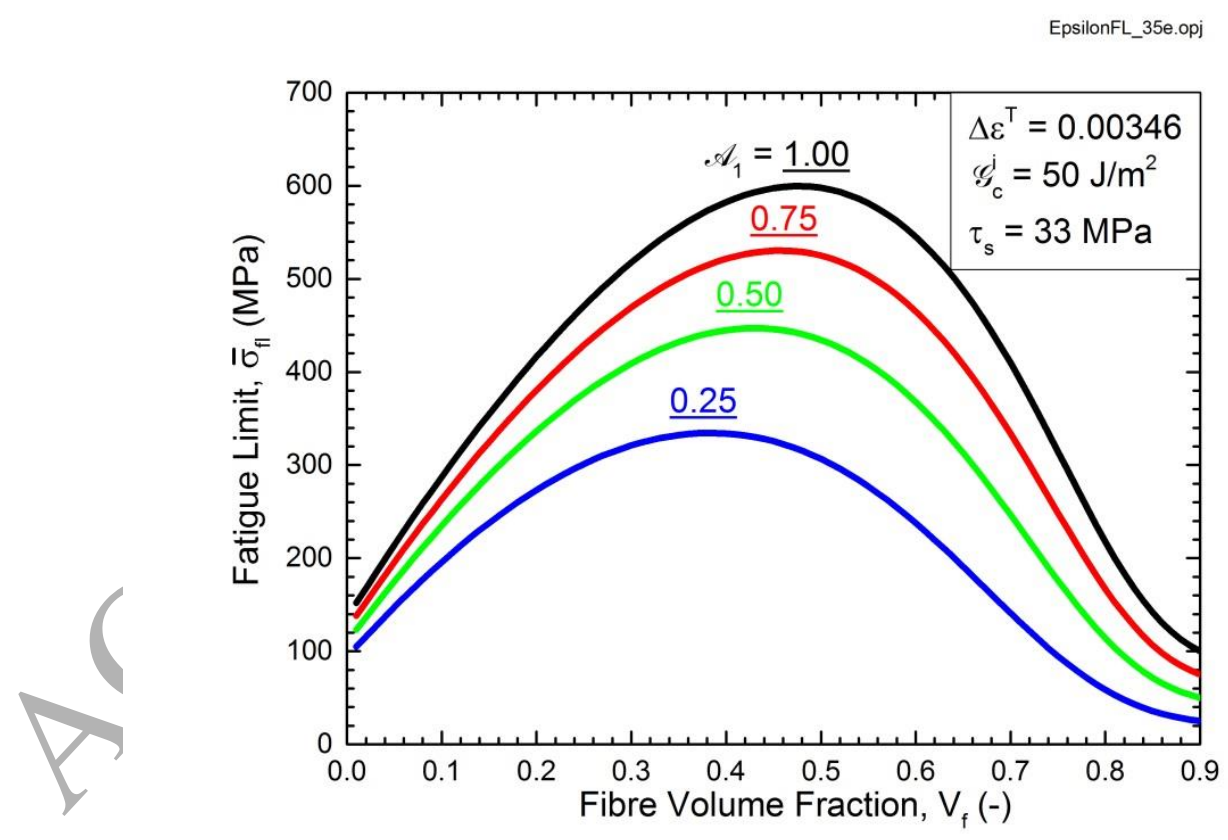

Fig. 5.5: The predicted fatigue limit expressed in terms of stress is shown as a function of fibre volume fraction for various values of the pre-factor $A_{1}$. 


\subsection{Effects of initial value of the interfacial frictional sliding shear stress}

Fig. 5.6 shows the predicted fatigue limit as a function of $\tau_{s}$ for various values of the pre-factor $A_{1}$. Clearly, with all other parameter held fixed, $\bar{\varepsilon}_{f l}$ increases monotonically with increasing value of $\tau_{s}$ and increasing value of $A_{1}$.

\subsection{Effects of interfacial fracture energy on fatigue limit}

We complete the parameter study by investigating the effects of $\mathrm{G}_{c}^{i}$. With the friction pre-factor held fixed, Fig. 5.7 shows $\bar{\varepsilon}_{f l}$ as a function of $\mathrm{G}_{c}^{i}$ for various values of $V_{f}$. $\bar{\varepsilon}_{f l}$ is highest for small values of $\mathrm{G}^{i}, \bar{\varepsilon}_{f l} \approx 2.3 \%$ for $\mathrm{G}^{i}$ close to zero, decreasing with increasing $\mathrm{G}_{c}^{i}$ to a near-constant value.

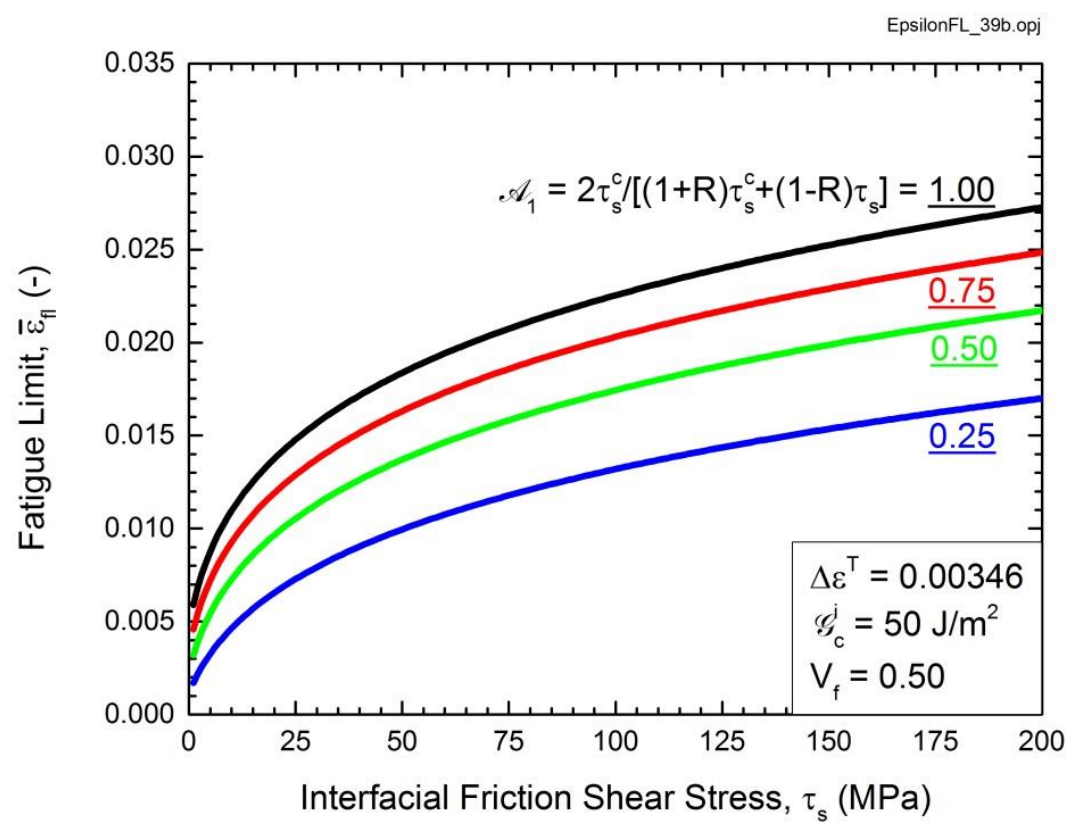

Fig. 5.6: The predicted fatigue limit shown as a function of interfacial frictional shear stress for various values of the pre-factor $A_{1}$. 


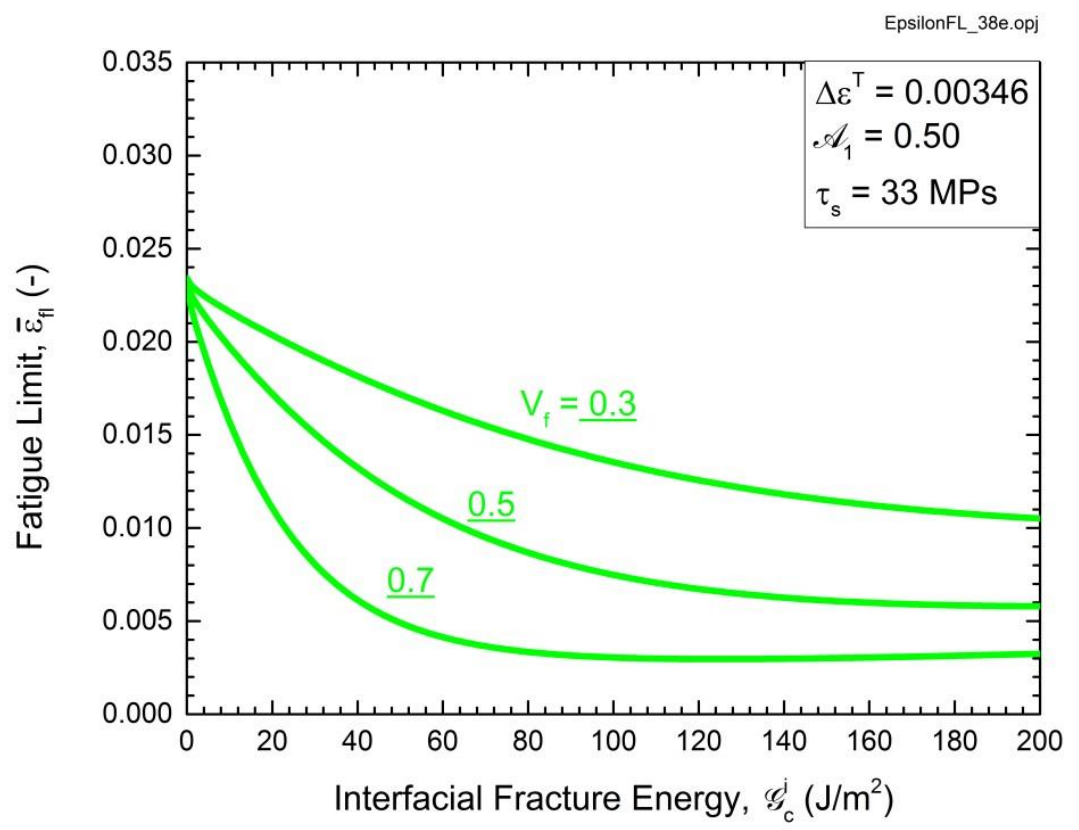

Fig. 5.7: The predicted fatigue limit shown as a function of interfacial fracture energy for three different values of the fibre volume fraction.

\section{Discussion}

\section{$\underline{6.1 . ~ C o m p a r i s o n ~ o f ~ m o d e l ~ a n d ~ e x p e r i m e n t a l ~ r e s u l t s ~}$}

We now compare model predictions with experimental results from the literature. The results of Mandell et al. (2003) suggest that the fatigue limit (run-out at $10^{10}$ cycles) was $1.2-1.5 \%$ for small strands consisting of only 45 fibres $\left(V_{f}=0.55, R=0.1\right.$ ) and $0.4-0.7 \%$ for unidirectional composites $\left(V_{f}=0.55, R=0.1\right)$. Data from the OptiDat database (Nijssen, 2012) suggest a fatigue limit for glass/epoxy (run out at $2 \times 10^{6}$ cycles, $V_{f}=0.53, R=0.1$ ) of about $0.5 \%$. The paper of Samborsky et al. (2008) includes data for lifetime of $10^{6}$ cycles $(R=0.1)$ for the fibre volume fraction ranging from 0.32 to 0.62 for a so-called DD series, which is a laminate consisting of $0^{\circ}$ and $\pm 45^{\circ}$ plies 
( $72 \%$ of the fibres are $0^{\circ}$, so we assume that the fatigue limit is not significantly influenced by the presence of the adjacked $\pm 45^{\circ}$ plies).

From the paper of Pupurs et al. (2013), the initial debond length of two single filament specimens made of glass/polyester tested at a maximum applied strain of $1.76 \%(R=0.1)$ were $\ell=10 \mu \mathrm{m}$ and $20 \mu \mathrm{m}$, respectively. After $\sim 10^{5}$ cycles, the corresponding values of the debond length, $\ell_{d}^{c}$, were 40 $\mu \mathrm{m}$ and $70 \mu \mathrm{m}$. Using the values of $E_{m}, E_{f}$, and $\tau_{s}$ from Table 1, we find from Eq. (3.42) $\tau_{s}^{c}=$ 19.4 MPa and 23.2 MPa. Using these values along with $R=0.1$, Eq. (4.12) gives $A_{1}=0.76$ and $A_{1}$ $=0.84$, respectively for the two specimens. Taking $\mathrm{A}_{1}=0.8$ as an average value and $V_{f}=0.55$, we read-off $\bar{\varepsilon}_{f l}=1.3 \%$ from Fig. 5.4 as the predicted fatigue limit. Given the uncertainty of the parameter determination - the parameters in Table 1 are from other studies - this prediction compares favourably with the experimentalvalues of run-out from Mandell et al. (2003) for the small strands $(1.2-1.5 \%)$ but not for the laminates $(0.4-0.7 \%)$. It is, however, likely that the there is some variation in the fibre volume fraction over the cross section of the laminates. If we assume that the laminates had fibre-rich regions with a local fibre volume fraction of, say 0.7 , the expected fatigue limit read off from Fig. $5.4\left(\mathrm{~A}_{1}=0.8, V_{f}=0.7\right)$ would be $\bar{\varepsilon}_{f l}=0.7 \%$. This prediction is within the range (0.4-0.7\%) found experimentally for the laminates by Mandell et al. (2003). As shown in Fig. 5.3, an increase in the R-ratio increases $A_{1}$ and thereby increases $\bar{\varepsilon}_{f l}$, see Fig. 5.4. This model prediction is in agreement with the trend observed experimentally for a quasiisotropic glass/polyester laminate (Mandell et al. (2003)). 
The experimental study of Partridge et al. (1989) showed that the fatigue limit of a unidirectional glass/epoxy composite made by pre-pregs (run out at $3 \times 10^{6}$ cycles, $R=0.05$ ) was $\bar{\varepsilon}_{f l}=0.91 \%$ for $V_{f}=47 \%$ and $\bar{\varepsilon}_{f l}=0.43 \%$ for $V_{f}=69 \%$, i.e. decreasing with increasing fibre volume fraction. This trend is consistent with the model prediction (Fig. 5.4). In the experiments by Partridge et al. (1989), specimen having $V_{f}=47 \%$, were made by inserting layers of epoxy films that resulted in resin rich interlaminar regions. Therefore, the reported fibre volume fraction in the fibre rich layers are likely to exceed $47 \%$. The overall trend of model prediction, $\bar{\varepsilon}_{f l}$ decreasing with increasing $V_{f}$ is also in agreement with the experimental data of the DD-series of Samborsky et al. (2008).

The experimental data for fatigue limit of the glass fibre composites investigated by Partridge et al. (1989), Mandell et al. (2003), the DD-series of Samborsky et al. (2008) and data from Nijssen (2012) are superimposed in Fig. 5.4. Error bars represent the estimated uncertainties. There is a reasonably good correspondence between the experiment data and the model predictions although the predictions (curves) are based on parameters not determined for the individual material systems. The experimental data for laminates with unidirectional glass fibres lie close to the curve corresponding to $A_{1}=0.25$. It is clear from Figs. 5.6 and 5.7 that other values of $\tau_{s}$ and $\mathrm{G}^{i}$ could lead to predictions closer to the experimental data. However, identification of parameters by fitting is not the aim of the present paper; we just wish to check if realistic material parameters from the literature (Table 1), determined from independent microscale experiments, give fatigue limit predictions that are comparable to experimental findings. Note also, that the data are plotted as a function of the average fibre volume fraction. But real composites are likely to have regions with locally higher fibre volume fractions. Had the higher fibre volume fractions been used to plot the 
experimental data, the points would have been shifted towards higher $V_{f}$, i.e., towards curves corresponding to higher $A_{1}$-values.

\subsection{Most important microscale parameters}

According to the model predictions for glass fibre composite with polymer matrix (Section 5), the most important composite parameters that control $\bar{\varepsilon}_{f l}$ are $V_{f}, \tau_{s}, \mathrm{G}^{i}$ and $\tau_{s}^{c}$. Notably, $\bar{\varepsilon}_{f l}$ is highest for vanishing $\mathrm{G}_{c}^{i}$. In comparison, the residual stress plays a minor role. The effect of $V_{f}$ is clearly seen in Fig. 5.2. As an example, with $\Delta \varepsilon^{T}=0, \bar{\varepsilon}_{f l}$ decreases from a relative high value, $\bar{\varepsilon}_{f l}$ $=1.9 \%$, for $V_{f}=0.3$ to $\bar{\varepsilon}_{f l}=0.7 \%$ at $V_{f}=0.7$. This is a quite significant decrease. While a value of $V_{f}$ of 0.3 may be too low for most practical engineering applications, a value of $V_{f} \approx 0.5$ corresponds to $\bar{\varepsilon}_{f l} \approx 1.4 \%$, which is probably realistie.

From Fig. 5.6, it follows that the model predicts that a higher value of the initial frictional sliding shear stress, $\tau_{s}$, will result in a higher yalue of $\bar{\varepsilon}_{f l}$. For instance, with the other parameters held fixed, the model predicts (for $\mathrm{A}_{1}=0.25$ ) $\bar{\varepsilon}_{f l}=0.73 \%$ for $\tau_{s}=25 \mathrm{MPa}$, with $\bar{\varepsilon}_{f l}$ increasing to 1.0 $\%$ for $\tau_{s}=50 \mathrm{MPa}$. Furthermore, as shown in Fig. 5.3, if the interfacial shear stress changes (decreases) significantly during repeatable forward and reverse slip, the pre-factor $A_{1}$ given by (4.12) takes a value below unity which leads to a lower fatigue limit. Thus, a higher fatigue limit would be obtained if the decrease in the fibre/matrix sliding friction was reduced or avoided.

The results in Fig. 5.7 indicate that for $V_{f}=0.5, \bar{\varepsilon}_{f l}$ can be enhanced above $1.5 \%$ by decreasing $\mathrm{G}^{i}$ below $10 \mathrm{~J} / \mathrm{m}^{2}$. It may appear counter-intuitive that a reduction in the fibre/matrix interface 
bonding can lead to an increase in the fatigue limit. However, according to the model, the fatigue limit is to a great extend controlled by the K-field of the debond crack tip. A reduction in the interfacial fracture energy reduces the stress intensity at the debond crack tip and thus the stress induced to the neighbouring fibres, $\hat{\sigma}_{f}^{K}$, see eq. (4.1).

\subsection{Implication for materials development}

The result, $\mathrm{G}_{\text {nin }}^{i}=\mathrm{G}^{i}$ (so that $\Delta K_{I I}=0$ and thus $\Delta K_{I I}<\left(\Delta K_{I I}\right)_{t h}$ ), eq. (3.24), infers that the fatigue damage evolution is not controlled by Paris-Erdogan fatigue crack growth of the fibre/matrix interface, neither for the single fibre fragmentation test nor for a fibre composite materials where the dominating fatigued damage mechanisms is the Gamstedt-Talreja mechanism. Instead, the progressive debonding can be attributed to a decreasing $\tau$.

On the other hand, the result, $\mathrm{G}_{\text {min }}^{i}=\mathrm{G}^{i}$, Eq. (3.23), implies that the debond crack tip is always loaded just to the level at which static debond crack propagation can occur. Therefore, a potential risk is that progressive debonding could occur by sub-critical crack growth along the fibre/matrix interface, e.g. being affected by exposure to humidity - it is well known that glasses are susceptible to stress corrosion cracking (Weiderhorn, 1967). The long term ability of the fibre coatings to protect glass fibres is thus crucial.

The results of the/micromechanical model suggest that it is possible to change (increase) the fatigue limit of unidirectional fibre composites by proper design of the fibre/matrix interface. The major suggestions for designing unidirectional composites with a higher fatigue limit are (i) to avoid a (local) high fibre volume fraction, (ii) ensure a low interfacial fracture energy (below $10 \mathrm{~J} / \mathrm{m}^{2}$ ), (iii) have a high initial interfacial frictional sliding shear stress and (iv) aim to avoid that the interfacial frictional sliding shear stress decreases during repeated forward and reverse slip. The finding of this 
paper could imbue the research field in interface control to put more weight on controlling the interfacial sliding friction and wear of asperities roughness of surfaces of fibres than on the chemistry to enhance the fracture energy of the fibre/matrix interface.

The models presented in this paper should be useful for experimentalists. As explained in Section 6.1, the initial values of the interfacial frictional sliding shear stress, $\tau_{s}$ can be determined from analysis of single filament specimens where the debond length is recorded as a function of a monotonically increasing applied stress. Next, by measurement of limit value of the debond length of single filaments subjected to cyclic loading, $\ell_{d}^{c}$, eq. (3.42) gives $\tau_{s}^{c}$. Knowing the R-ratio, the parameter $A_{1}$ can then be calculated from (4.12). Now all parameters needed are determined and the model can be applied for prediction of the fatigue limit, either by solving (4.21) using the appropriate material properties, or by the use of curves as those presented in Figs. 5.4 to 5.7. In addition, in case it is possible to measure the fibre openings experimentally during cyclic loadingunloading experiments, the equations for the stress-openings described in Appendix A can be used to back-calculate the interface parameters, e.g. measure the frictional sliding shear stress decrease during repeated forward-reverse interfacial slip.

\subsection{On the prediction of zero stress intensity factor range}

A numerical model was made in order to verify that $\Delta K_{I I}=0$ for the problem in Fig. 2.1 during unloading and reloading. Details of the finite element model and the major findings are described in Appendix B. In brief, the model results confirm that indeed $\Delta K_{I I}=0$ in the case there is sticking friction ahead of the debond crack tip. 
The result $\Delta K_{I I}=0$ during macroscopic unloading, should not be taken as a surprise. During the macroscopic unloading, there is unloading everywhere at the fibre/matrix level, but in the sticking zone and ahead of the crack tip (i.e., for $\ell_{s}^{u}<z$ ), the fibre and matrix undergo identical strain changes. It is well-known from 2-dimensional linear-elastic problems (Suo and Hutchinson, 1990; Suo, Bao, Fan, 1992) that uniform (i.e. $z$-coordinate independent) tensile and bending strain fields do not create a singular crack tip stress field and thus results in zero energy release rate. Thus, adding such a stress field should leave the crack tip energy release rate unchanged.

Also, from a physical point of view, it can be argued that the sticking friction (non-slip) zone at the crack tip should result in unchanged $K_{I I}$. For a Mode II crack, the near-tip displacements scales linearly with the Mode II stress intensity factor (Kanninen and Popelar, 1985). Thus, no change in the crack tip displacement field is equivalent to no change in $K_{I I}$. This is the situation of a sticking friction zone at the crack tip during cyclic loading: There occurs no further displacement between the fibre and matrix during cyclic loading and reloading.

The finding that $\Delta K_{I I}=0$ when there is a zone with sticking frictional shear stress at the debond crack tip is in contrast to the friction-free problem for which $\Delta K_{I I}$ is non-zero (Pupurs et al., 2013; Alves and Pimenta, 2018). Disregarding friction, the problem becomes linear, and the stress field (and thus the Mode II stress intensity factor, $K_{I I}$ ) scales linearly with the applied stress.

\subsection{Limitation of the model}

The present model is a traditional shear-lag model, for which it is known that they are only accurate for debond length exceeding a few fibre radii (e.g., $\ell_{d} / r>5$ ) - see e.g. Hutchinson and Jensen 
(1990) and Sørensen (2017) for more thorough discussions. Similar requirements are expected for the unloading and reloading slip lengths, $\ell_{s}^{u c} / r>5$ and $\ell_{s}^{l c} / r>5$. Thus, the equations for opening displacements described in Appendix A are most accurate for the end-states, e.g. for values of openings at the maximum and minimum applied strain values where the requirements of long slip lengths are expected to be fulfilled.

The stress transfer analysis (Part I) has similarities with earlier models of multiple matrix cracking (McMeeking and Evans, 1990; Cho et al., 1991; Pryce and Smith, 1993; Keith and Kedward, 1995; Ahn and Curtin, 1997). The major differences are (a) that the roles of fibre and matrix are switched, (b) our model includes two different values of the interfacial frictional sliding shear stress (one for the first forward slipping region, another for the part of the interface that experiences repeated forward/reverse slip) and (c) our model include crack tip débonding. In the earlier models, that did not include fibre/matrix debonding, the stresses in fibre and matrix were both continuous at the end of the slip zone. Shear-lag models that include a crack tip (energy release rate) possess a stress jump at the crack tip (Hutchinson and Jensen, 1990), as also seen in Fig. 2.3.

The numerical model (Appendix B) that includes Poisson's effects and models the slipping interface in terms of Coulomb friction was used to check the accuracy of the analytical model. It was found that for a smooth fibre/matrix interface, the interfacial frictional shear stress was constant along the slip zone with Poisson's ratio's of the fibre and matrix both being zero, $v_{f}=v_{m}=0$. For non-zero values of $v_{f}$ and $v_{m}$, the radial normal stress and the interfacial shear stress during slip vary as a function of $z$-position (typically, \pm 20 to $40 \%$ ) within the slip zone and applied stress level. The assumption of a constant interfacial sliding shear stress is thus a fair approximation for smooth fibres. But, as pointed out by Marshall et al. (1992), the sliding mechanics of real fibre/matrix 
interfaces is more complicated than Coulomb friction; roughness asperities at the fibre surfaces significantly influence the sliding mechanics. In their experimental study, they found that the interfacial frictional shear stress behaved differently from the response expected of a smooth interface governed by a Coulomb friction law. They found that the interfacial sliding resistance was constant along the debonded region of interface. They suggested that effects of the roughness asperities and the Poisson's effects cancel locally over the entire sliding region. This leads us to conclude that a constant frictional sliding shear stress is a good representation of the sliding behaviour of fibre/matrix interfaces.

Most importantly, the results from the finite element model (Appendix B) confirm that during unloading (Time Step 2), the stress transfer along the debonded interface with sticking friction remains "frozen", an assumption made for the sticking-friction zones in the analytical model (Sections 3.2 to 3.4 ).

In the present model, the matrix material is modelled as being a linear-elastic solid. This is an important simplification for application for polymer matrix composites, since polymers may possess visco-elastic or visco-plastic properties which could influence their long-term behaviour. For instance, the stress state at the crack tip can be relaxed by creep. Creep could develop differently for high and low load frequencies and thus influence the fatigue limit. However, creep might not necessarily change the basic debond crack arrest mechanism modelled in this paper (the Gamstedt-Talreja mechanisms). At the microscale, the failure stress and strain can be significantly higher than their macroscale counterparts (Zike et al., 2016). Also, at the microscale, composites often experience tri-axial stress state, which again may limit visco-plasticity. Further studies are required to uncovering effects of visco-elasticity and visco-plasticity on fatigue. 
In the present study the fracture process zone of the debond crack tip is modelled as small-scale so that it is taken to be embedded in a K-field. The solution used for estimating the stress induced to the surrounding fibres (Section 4.1 and Appendix C) is most accurate for $r^{*}<<r$ (i.e., close to the debond crack tip). For high fibre volume contents, the fibre spacing may be so small that the stress induced into the surface of the surrounding fibres may be influenced by details of the fracture process zone of the debond crack tip. A more detailed representation of the fracture process zone could be made by the use of a cohesive zone. Such analysis could explore how sensitive the stress in surrounding fibres is to e.g. details of the cohesive law.

In this study, the two main assumptions regarding the mechanical behaviour of the fibre/matrix interface are: (1) the cyclic debond growth rate can be represented with a Paris-Erdogan law with a threshold value and (2) the interfacial frictional sliding shear stress decreases to a lower value due to repeated forward/reverse slip during cyclic loading. The empirical Paris-Erdogan law has previously been used for describing cyclic crack growth along fibre matrix interface in composites (Pupurs et al., 2013; Alves and Pimenta, 2018) although there are no direct experimental evidence of its applicability for the problem of cyclic interface debonding. As mentioned, the crack growth rate has been found to decrease and even vanish for cyclic debonding of single fibers (Gamstedt and Talreja, 1999; Goutianos and Peijs, 2001; Pupurs et al., 2013). As shown in Section 3.2, this behaviour is consistent with the situation with a frictional sliding shear stress that decreases to a stationary value during cyclic loading, leading to a zone with sticking friction near the crack tip. It would thus be difficult to measure a Paris-Erdogan law for a debond crack tip with sticking friction. However, as the present model explains, with sticking friction, $\Delta K_{I I}$ becomes zero, so that only the assumption of zero crack growth for zero $\Delta K_{I I}$ has significance. 
As for the decrease in frictional shear stress, there are experimental evidence that wear of roughness asperities of fibres in ceramic matrix composites (Mackin et al., 1992; Marshall et al., 1992). This mechanism may also be active in other composite systems. For polymer matrix composites it can also be expected that plastic deformation may take place at roughness asperities of fibres (at the nanometre scale) and thus leads to lowering of the frictional sliding shear stress. Further experimental work, e.g. studying the changes in fibre end openings during cyclic loading, are needed to clarify this point.

Note that since the model is essentially a 1D model, it can be applied directly to anisotropic fibres such as carbon fibres with $E_{f}$ and $\alpha_{f}$ now being the Young's modulus and thermal expansion coefficient in the longitudinal (fibre) direction.

The assumptions used for establishing the analytical model in the present study (i.e. disregarding Poisson's effects of fibre and matrix, the choosing of a constant interfacial shear stress, e.g., as opposed to a Coulomb friction) are taken deliberately to enable the development of simple analytical solutions that are easy to solve and apply. The complexity and accuracy of micromechanicalmodels should be balanced to match the accuracy of what is possible to obtain from state-of-the-art of the experimental methods.

\subsection{Other damage mechanisms}

The present model implicitly assumes that as the fibre fractures, debonding occurs at the fibre matrix interface. However, it is also possible that the crack does not deflect along the fibre/matrix interface. According to He and Hutchinson (1989), crack deflection along an interface will only 
occur providing that the fracture energy of the interface is sufficiently low relative to the fracture energy of the matrix material. Simulations based on cohesive zone modelling show, however, that whether crack deflection takes place along an interface depends on both the fracture energy and peak-traction values of the cohesive laws of the fibre/matrix interface and cohesive laws of the matrix material (Parmigiani and Thouless, 2007).

The model developed in the present paper concerns the situation where isolated fibre fáilure (and fibre/matrix debonding) is the first and only fatigue damage mechanism, following the experimental observations of Gamstedt and Talreja (1999). It is, however, plausible that other damage mechanisms may develop, in particularly at higher strain values, such as cracking of the matrix (Talreja, 1981). Such other fatigue damage mechanisms may develop progressively at a strain value than is lower than the value of $\bar{\varepsilon}_{f l}$ predicted in the present paper. Also, for composite laminates consisting of multidirectional plies or non-crimp fabrics (rowing's with stitched backing bundles that are oriented off the tensile direction), the development of transverse cracks can lead to the growth of transverse cracks into the unidirectional plies and can thus induce fatigue damage (fibre failure) in nearby plies (Jamison et al., 1984; Zangenberg et al., 2013), either by fibre failures at the matrix crack tip or in the crack wake. Those situations are beyond the assumptions of the present model and should thus be analyzed by other models.

\section{Concluding remarks}

The micromechanical model developed in the present paper for the Gamstedt-Talreja fatigue mechanism predicts that the stress induced to neighbouring fibres increases with increasing 
interface fracture energy and decreasing fibre spacing; this is in complete agreement with the experimental findings of van den Heuvel et al. (1997).

The model shows that in case there is sticking friction at the fibre/matrix interface at the tip of a debond crack of a broken fibre, there will be no change in the crack tip stress intensity factors during cyclic loading. Cyclic crack growth of the debond crack will then stop. Thus, the model predictions explain the observations of debond crack growth arrest made by Gamstedt and Talreja (1999).The presence of friction along the fibre/matrix interface thus opens the possibility that a fatigue limit, defined from the stability of damage evolution of a single broken fibre, can exist. The model predicts that the fatigue limit will increase for higher R-ratio (in the range of $0<R<1$ ). This prediction is consistent with the experimental findings of Mandell et al. (2003).

According to the model, a fatigue limit can be increased by increasing the initial value of the interfacial sliding shear stress, by minimizing the decrease in the interfacial sliding shear stress during repeatable cyclic slip, decreasing the fibre volume fraction and decreasing the interfacial fracture energy. The effects of residual stresses are insignificant for fibre volume fractions higher than about $50 \%$. Model predictions of the fatigue limit made for a glass/epoxy composites compare favourably with experimental findings.

\section{Appendix A}


Relations between $\bar{\sigma}$ and $\delta$ can be used to identify interface parameters from experiments. In this appendix we present equations for the half fibre opening displacement, $\delta$, under cyclic loading. Mathematically, $\delta$ is calculated as

$$
\delta(\bar{\sigma})=\int_{0}^{\ell_{d}^{c}}\left(\frac{\sigma_{m}^{-}(z)}{E_{m}}-\frac{\sigma_{f}^{-}(z)}{E_{f}}-\Delta \varepsilon^{T}\right) d z,
$$

where is $\sigma_{m}^{-}(z)$ and $\sigma_{f}^{-}(z)$ are stresses in the debonded zone, and $\Delta \varepsilon^{T}$ is the mismatch strain. The notation $\delta(\bar{\sigma})$ in (A-1) is used to indicate that $\delta$ is a function of $\bar{\sigma}$.

The result of the first load cycle (in which $\ell_{d}^{c}$ is replaced by $\ell_{d}$ ) is given by (3.7). For the first unloading, the integral (A-1) has to be split in two parts, since different equations apply for the different slip zones. The relevant equations for $\sigma_{m}^{-}(z)$ and $\sigma_{f}^{-}(z)$ are (3.9) for the reverse slip zone $\left(0<z<\ell_{s}^{u}\right)$ and (3.11)-(3.12) for the sticking zone $\left(\ell_{s}^{u}<z<\ell_{d}\right)$. The result is

$$
\frac{\delta(\bar{\sigma})}{r}=\frac{\delta\left(\bar{\sigma}_{\max }\right)}{r}-\frac{1}{4} \frac{\left.E_{f}\right\rangle}{E_{c}} \frac{\left(\bar{\sigma}_{\max }-\bar{\sigma}\right)^{2}}{\left(1-V_{f}\right) E_{m}\left(\tau_{s}^{u}+\tau_{s}\right)},
$$

where $\delta\left(\bar{\sigma}_{\max }\right)$ is the opening displacement of the first loading at $\bar{\sigma}_{\max }$, i.e., obtained by setting $\bar{\sigma}=\bar{\sigma}_{\max }$ in $(3.7)$

For cycling reloading we need to consider three different slip zones. The stresses $\sigma_{f}^{-}(z)$ and $\sigma_{m}^{-}(z)$ are given by eq. (3.25) for $0<z<\ell_{s}^{l c}$ where $\ell_{s}^{l c}$ is given by (3.26), and by (3.28) and (3.29) for $\ell_{s}^{l c}<z<\ell_{s c}^{l c}$, where $\ell_{s c}^{l c}$ is given by (3.27). For $\ell_{s c}^{l c}<z<\ell_{d}^{c}$, the stresses $\sigma_{f}^{-}(z)$ and $\sigma_{m}^{-}(z)$ are given by (3.29)and (3.30). Inserting these equations into (A-1) and performing the integration gives 


$$
\begin{gathered}
\frac{\delta(\bar{\sigma})}{r}=\left\{\frac{\bar{\sigma}}{\left(1-V_{f}\right) E_{m}}-\Delta \varepsilon^{T}\right\}\left(\frac{\ell_{s}^{l c}}{r}\right)+\frac{E_{c}}{\left(1-V_{f}\right) E_{m}} \frac{\tau_{s}^{c}}{E_{f}}\left\{\left(\frac{\ell_{s c}^{l c}}{r}\right)^{2}-2\left(\frac{\ell_{s}^{l c}}{r}\right)^{2}\right\}, \\
+\left\{\frac{\bar{\sigma}_{\min }}{\left(1-V_{f}\right) E_{m}}-\Delta \varepsilon^{T}\right\}\left\{\left(\frac{\ell_{s c}^{l c}}{r}\right)-\left(\frac{\ell_{s}^{l c}}{r}\right)\right\}+\frac{\Delta u\left(\ell_{s c}^{l c}\right)}{r}
\end{gathered}
$$

where $\Delta u\left(\ell_{s c}^{l c}\right)$ is the displacement difference of the fibre and matrix at the position $z=\ell_{s c}^{l c}$ :

$$
\frac{\Delta u\left(\ell_{s c}^{l c}\right)}{r}=\left[\frac{\bar{\sigma}_{\min }}{\left(1-V_{f}\right) E_{m}}+\frac{1}{2} \frac{\Delta \bar{\sigma}_{c}^{c}}{\left(1-V_{f}\right) E_{m}}\left(1+\frac{\tau_{s}}{\tau_{s}^{c}}\right)-\Delta \varepsilon^{T}\right]\left\{\frac{\ell_{d}^{c}}{r}-\frac{\ell_{s c}^{u c}}{r}\right\}-\frac{E_{c}}{\left(1-V_{f}\right) E_{m}} \frac{\tau_{s}}{E_{f}}\left\{\left(\frac{\ell_{d}^{c}}{r}\right)^{2}-\left(\frac{\ell_{s c}^{u c}}{r}\right)^{2}\right\}
$$

For cycling unloading we also need to consider three different zones. The stresses $\sigma_{f}^{-}(z)$ and $\sigma_{m}^{-}(z)$ are given by eqs. (3.35)-(3.36) for $0<z<\ell_{s}^{u c}$, while $\sigma_{f}^{-}(z)$ and $\sigma_{m}^{-}(z)$ are given by (3.39)(3.40) for $\ell_{s}^{u c}<z<\ell_{s c}^{u c}$ and by (3.30) and (3.31) for $\ell_{s}^{u c}<z<\ell_{d}^{c}$. Inserting into (A-1) leads to:

$$
\begin{gathered}
\frac{\delta(\bar{\sigma})}{r}=\left\{\frac{\bar{\sigma}}{\left(1-V_{f}\right) E_{m}}-\Delta \varepsilon^{T}\right\}\left(\frac{\ell_{s}^{U c}}{r}\right)+\frac{E_{c}}{\left(1-V_{f}\right) E_{m}} \frac{\tau_{s}^{c}}{E_{f}^{\prime}}\left\{-\left(\frac{\ell_{s c}^{l c}}{r}\right)^{2}+2\left(\frac{\ell_{s}^{u c}}{r}\right)^{2}\right\} . \\
+\left\{\frac{\bar{\sigma}_{\max }}{\left(1-V_{f}\right) E_{m}}-\Delta \varepsilon^{T}\right\}\left\{\left(\frac{\ell_{s c}^{l c}}{r}\right)-\left(\frac{\ell_{s}^{u c}}{r}\right)\right\}+\frac{\Delta u\left(\ell_{s c}^{l c}\right)}{r}
\end{gathered}
$$

In summary, first the relevant cyelic slip lengths are determined as follows: $\ell_{s}^{l c}$ is given by (3.26), $\ell_{s c}^{l c}$ by (3.27) and $\ell_{d}^{c}$ by (3.33) or (3.34). Then, $\Delta u\left(\ell_{s c}^{l c}\right)$ can be determined from (A-4) and $\delta(\bar{\sigma})$ can be determined during cyclic reloading from (A-3). With $\ell_{s}^{u c}$ is given by (3.37) and $\ell_{s c}^{u c}$ by (3.38), $\delta(\bar{\sigma})$ can be determined during cyclic unloading by (A-5).

\section{Appendix B}

$\underline{\text { B.1. Details of numerical (finite element) model }}$ 
An axisymmetric finite element model is used to check the prediction, e.q. (3.24), of the analytical model that $\Delta K_{I I}=0$ in case there is sticking friction ahead of the debond crack tip. Fibre and matrix are modelled as isotropic elastic materials with $E_{f}=70 \mathrm{GPa}$ and $E_{m}=3 \mathrm{GPa}$; the Poisson's ratio of fibre and matrix were set to the same value, $v_{f}=v_{m}$. The fibre volume was $50 \%$ and the fibre radius was $8 \mu \mathrm{m}$. Fig. B. 1 shows the two-dimensional axisymmetric finite element used.

The model includes a debond zone of fixed length, $\ell_{d}$ and the fibre is traction-free at the location of the fibre break, $z=0$. Along the debonded surfaces, the frictional stress transfer between fibre and matrix is controlled by Coulomb friction, modelled by contact surfaces. The model was made using the commercial finite element program Abaqus (2017), using reduced integration, 8-node quadratic axisymmetric elements.

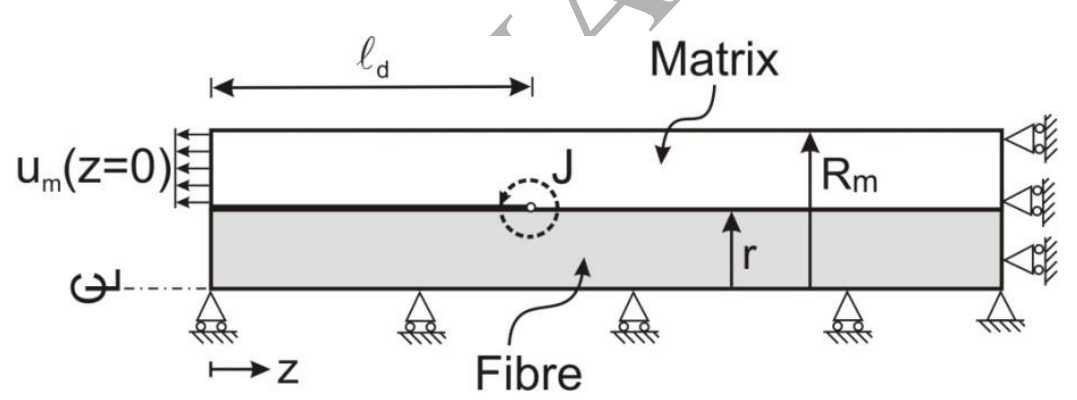

AxisymmetricFEM_BC_1b.cdr

Fig. B.1: Geometry and boundary conditions of the axisymmetric finite element model of a single broken fibre embedded in a matrix cylinder. The length of the debond is fixed at $\ell_{d}$.

First (Time Step 0), a temperature change was applied in the model. During this step, the nodes of the matrix-end $(z=0)$ were constrained to have the same displacement in the $z$-direction to fulfil the symmetry-condition at $z=0$. In the next step (Time Step 1), a traction was applied incrementally at a reference point $(z<0)$. The displacements of the matrix nodes at $z=0$ were 
prescribed to have the same displacement $u_{m}$ as the reference point. The applied traction caused the far field stress to reach $\bar{\sigma}_{\max }$ while enabling frictional slip along the interface with contact ( $0 \leq z \leq \ell_{d}$ ). Next (Time Step 2), the applied stress is reduced to $\bar{\sigma}_{\min }$ (unloading). The R- ratio was equal to 0.1 . For the investigation of the crack tip stress intensity under cyclic slip along the fibre/matrix interface, two additional loading-unloading cycles (Time Steps 3 and 4) were added.

Note that the problem solved with the finite element model does not accurately represent the real problem of Fig. 2.1. For the real problem, the debond length increases with the first mechanical loading in accordance with (3.5), whereas the debond length is fixed in the finite element model. However, for both the real problem and the finite element model, the subsequent unloading and the next loading-unloading cycles (corresponding to Time Steps 2 to 4 in the finite element simulation) occur under fixed debond length.

During the loading and unloading steps, the energy release rate $\mathrm{G}^{i}$ is computed numerically using the $\mathrm{J}$ integral along rings around the crack tip. For a traction-free crack, the $\mathrm{J}$ integral values should be identical for all rings due to the path independency of the $\mathrm{J}$ integral (Rice, 1968). However, since there is friction in the debond zone, the J-integral does not give the same value for all rings. For this reason, the J-integral results presented here were taken from the same contour (a near-circular path around the crack tip with radius of ring $0.04 r$ ) for all cases. Also, the finite element mesh close to the crack tip was identical for all the cases.

\section{B.2. Fibre stress along interface}


First we investigate the evolution of the stress in the fibre using the finite element model. Fig. B.2 compares the stress in the fibre (for $v_{f}=v_{m}=0$ ) from the finite element model and the analytical model for two situations, at the end of first loading and at the end of first unloading. Results from the analytical model (Eq. (3.6), (3.9) and (3.11) are shown along with stresses from the finite element model (along the centre of the fibre and along fibre/matrix interface). In this example, a thermal expansion is introduced in the radial direction only (i.e., no axial thermal expansion) in order to create a normal pressure along the debonded interface. The thermal expansion coefficients of fibre and matrix in the radial direction were set to $10^{-6} /{ }^{\circ} \mathrm{C}$ and $10^{-3} /{ }^{\circ} \mathrm{C}$ respectively and the temperature changes was set to $-250{ }^{\circ} \mathrm{C}$. The friction coefficient was set to be 0.3 . In the analytical model, values of $\tau_{s}=19.5 \mathrm{MPa}$ and $\Delta \varepsilon^{T}=0$ are used.

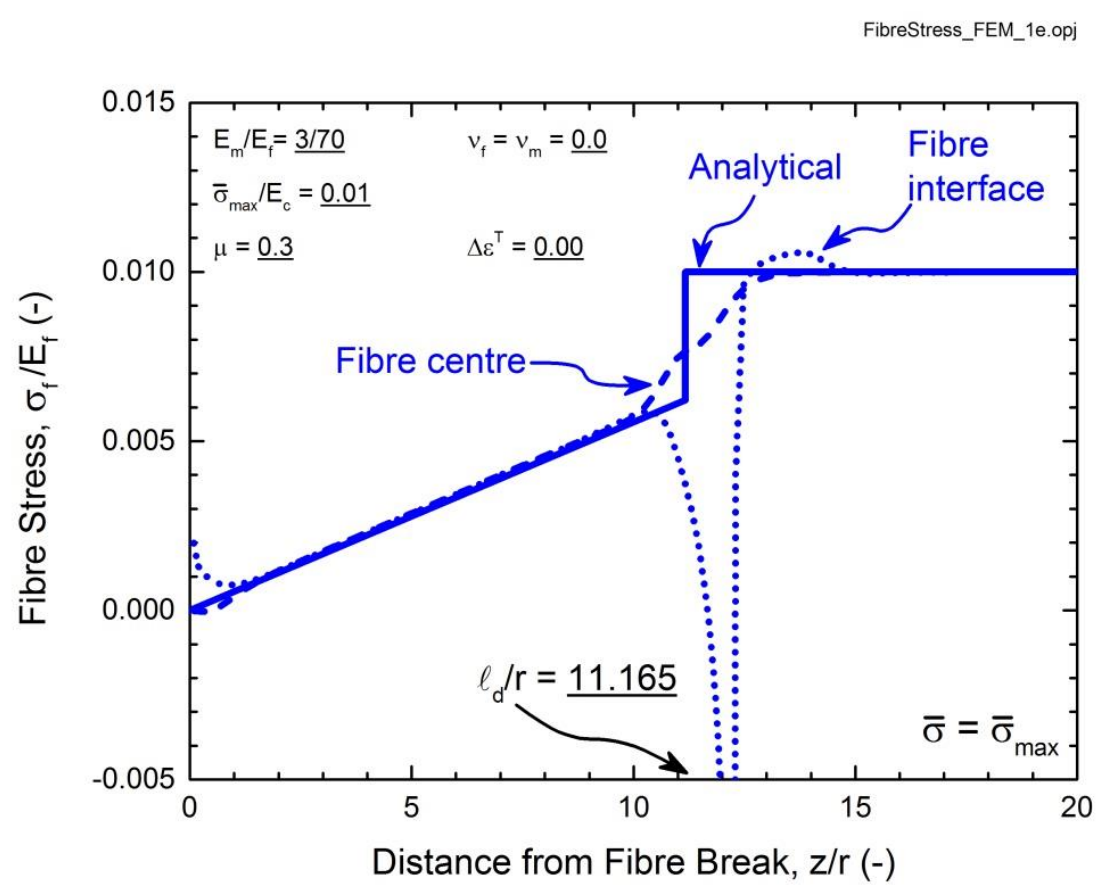




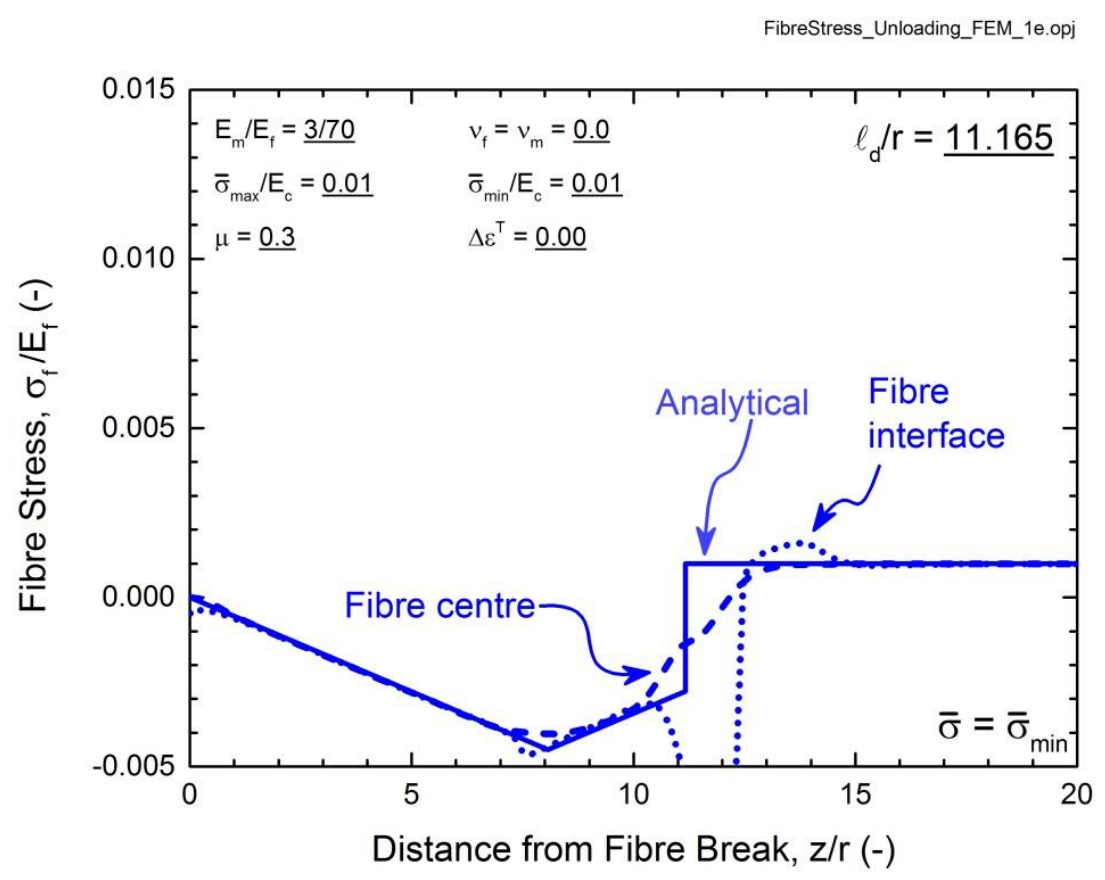

Fig. B 2: The fibre stresses from the finite element model and the analytical model (solid curve) are shown as a function of position along the fibre from the broken end for (a) $\bar{\sigma}=\bar{\sigma}_{\max }$ (end of first loading) and (b) for $\bar{\sigma}=\bar{\sigma}_{\text {min }}$ (end of first unloading). Results of the finite element model are shown for stresses in the middle of the fibre (dashed curve) and at the fibre surface along the fibre/matrix interface (dotted curve).

Fig. B.2 (a) corresponds to $\bar{\sigma}=\bar{\sigma}_{\max }$ (i.e., at the maximum applied stress of the first loading, Time $=1.0$ ) and Fig. B.2 (b) is for $\bar{\sigma}=\bar{\sigma}_{\text {min }}$ (minimum applied stress at the end of the first unloading, Time $=2.0$ ). It is seen in Fig. B.2 (a) that the stresses of the finite element model closely follow the stress of the analytical model except near the broken fibre end $(0<z / r<1)$ and around the debond crack tip $(10<z / r<15)$. The fibre stress at the fibre/matrix interface displays a strong effect of the singularity at the crack tip whereas the stress in the fibre centre changes more gradual. Fig. B.2 b) shows the fibre stresses for $\bar{\sigma}=\bar{\sigma}_{\min }$. Again, the stresses for the finite element model 
follow that of the analytical model closely. For $z / r<8.0$, there is reverse slip and the fibre stress becomes negative. For $z / r>8.0$ there is sticking friction and the stress profiles follow those obtained for $\bar{\sigma}=\bar{\sigma}_{\max }$ except for a constant different of $\left(\bar{\sigma}_{\max }-\bar{\sigma}_{\min }\right) E_{f} / E_{c}$ in accordance with (3.10). This confirms the assumption made in Section 3.2 that the stress profile is "frozen". Therefore, overall, there is a fairly good correspondence between predictions of the analytical and the more accurate numerical model.

\section{B3. Calculation of crack tip energy release rate value}

Next in Fig. B.3, the value of $\mathrm{G}^{i}$ at the crack tip is shown for two different fixed debond lengths. In these simulations, fibre and matrix were taken to be isotropic with $v_{f}=v_{m}=0.2$. The thermal expansion coefficient of the fibre and matrix were set to $10^{-6} /{ }^{\circ} \mathrm{C}$ and $10^{-5} /{ }^{\circ}$. The magnitude of the temperature change was adjusted to be $-335^{\circ} \mathrm{C}$, such that the far-field compressive fibre residual stress (normal stress in the radial direction, normal to the fibre/matrix interface) in the bonded zone was equal to $10 \mathrm{MPa}$. The friction coefficient was set to 0.5 . A value of $\Delta \varepsilon^{T}=0.3015 \%$ was used in the analytical model.

For short debond length, $\ell_{d} / r=11.165$, the change of $\mathrm{G}^{i}$ between cyclic loading (Time Step 3) and unloading (Time Steps 2 and 4) is significant, i.e. $\mathrm{G}_{\max }^{i}>\mathrm{G}_{\min }^{i}$. In the first unloading, $\mathrm{G}^{i}$ is initially constant (Time between 2.0 and 2.9) but starts to decrease (after Time $=2.9$ ). Checking the evolution of the interfacial shear stress it was found that the slip length reaches the crack tip (the sticking zone vanishes) at Time $=2.9$. The decrease in $\mathrm{G}^{i}$ can result in $\left|\Delta K_{I I}\right|>\left(\Delta K_{I I}\right)_{t h}$ leading to cyclic growth of the debond crack in accordance with (2.1). Likewise, for the reloading, $\mathrm{G}^{i}$ is constant and the new forward slip length is shorter than the debond length for Time between 3.0 and 
3.5. For the final unloading, $\mathrm{G}^{i}$ is constant in the time interval (Time between 4.0 and 4.8) where the reverse slip zone is shorter than the debond length.

When the debond length is longer, $\ell_{d} / r=18.5, \mathrm{G}^{i}$ remains constant throughout Time Steps 2 to 4 , i.e., $\mathrm{G}_{\max }^{i}$ is equal to $\mathrm{G}_{\text {min }}^{i}$ so that $\Delta K_{I I}=0$. Then, $\Delta K_{I I}<\left(\Delta K_{I I}\right)_{t h}$, so that no further cyclic crack growth can occur. In this case, the numerical results show that the slip during unloading and reloading is confined to a region away from the crack tip. A sticking zone is then always present at the crack tip. Thus, the numerical results confirm that $\Delta K_{I I}=0$ in the case that there is sticking friction at the crack tip, verifying Eq. (3.24).

J_integralDebondLength_2d.opj

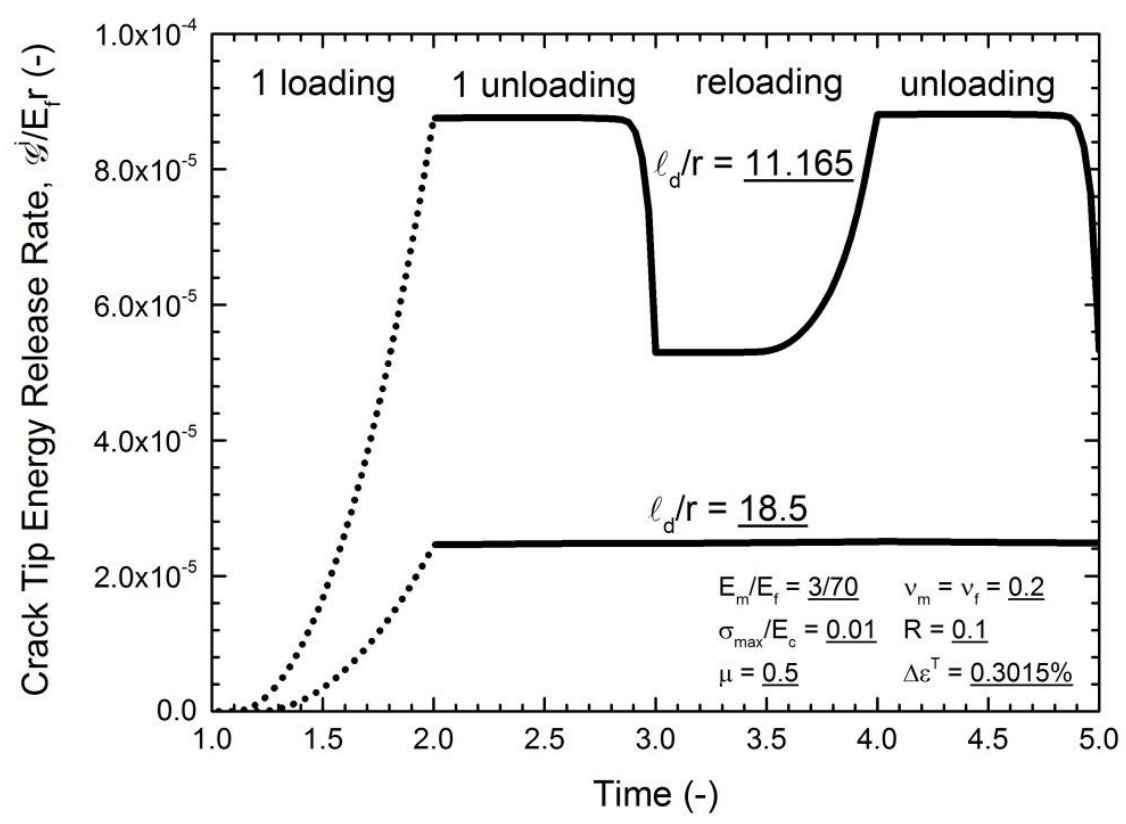

Fig. B.3: Results are obtained from finite element modelling: Normalized crack tip energy release rate is shows as a function of time during the two first loading-unloading cycles for two different debond lengths. 


\section{Appendix C. Stress in neighbouring fibres}

We will use a 2D plane strain model to estimate the highest maximum stress, denoted $\hat{\sigma}_{f}^{K}$

(superscript $K$ indicates the stress originating from the K-field), in the neighbouring fibres (located a distance $d^{*}$ away from a broken fibre) that is induced by the elastic crack tip stress field of a debond crack as it moves past a material point. As a first simple approximation, we will ignore the elastic mismatch and the mathematical complexities of bi-material crack tip stress fields (Rice, 1988), see Fig. 4.1. We estimate the maximum fibre stress, $\hat{\sigma}_{f}^{K}$, from the strain field of an elastic homogeneous material, in this case the matrix material that transfers the stresses from the broken and debonded fibre to the neighbouring fibre. The strain at a given material point is obtained from the Mode II displacement field (Kanninen and Popelar, 1985) as

$$
\varepsilon_{11}\left(r^{*}, \theta\right)=\frac{\left(1+v_{m}\right)}{8 \pi} \frac{K_{I I}}{E_{m}} \sqrt{\frac{2 \pi}{r^{*}}}\left[-(2 \kappa+1) \sin \frac{\theta}{2}-\sin \frac{5 \theta}{2}\right]
$$

where $r^{*}$ is the radial distance from the crack tip and $\theta$ is the angle measured in mathematical positive (counter clockwise) direction from the crack tip, $v_{m}$ is the Poisson's ratio of the matrix, $K_{I I}$ the Mode II stress intensity factor and $K$ is the Kolosov constant given by

$$
\kappa= \begin{cases}(3-v) /(1+v) & \text { for plane stress } \\ 3-4 v & \text { for plane strain }\end{cases}
$$

Assuming that the strain component $\varepsilon_{11}$ is continuous along the fibre/matrix interface at the neighbouring fibre, we can estimate the stress in the surface of the fibre from the strain using Hooke's law

$$
\sigma_{f}^{K} \approx E_{f} \varepsilon_{11}\left(r^{*}, \theta\right)
$$


where $E_{f}$ is the Young's modulus of the fibre. In (4.3) we have ignored Poisson's effect for simplicity. We wish to determine the angle $\theta$ that maximizes $\varepsilon_{11}$. Assume that the fibre spacing is $d^{*}$. First from pure geometry (Fig. 4.1):

$$
r^{*} \sin \theta=d^{*}
$$

Inserting $\kappa$ for plane strain from (C.2) and $r^{*}$ from (C.4) into (C.1) we obtain

$$
\varepsilon_{11}=\frac{\left(1+v_{m}\right)}{\sqrt{32 \pi}} \frac{K_{I I}}{E_{m} \sqrt{d^{*}}} g(\theta)
$$

where the function $g(\theta)$ is given by

$$
g(\theta)=\sqrt{\sin \theta}\left[\left(-7+8 v_{m}\right) \sin \frac{\theta}{2}-\sin \frac{5 \theta}{2}\right] .
$$

In order to find the maximum value of $\varepsilon_{11}$, denoted $\left.\varepsilon_{11}\right|_{\max }$, we find the maximum value of $g(\theta)$ within the range of $0^{\circ}<\theta<180^{\circ}$ as

$$
\left.g(\theta)\right|_{\max } \cong g\left(\theta \approx 45.6^{\circ}\right)=-3.356
$$

when $v_{m}=0.3$. Inserting $v_{m}=0.3$ and $\left.g(\theta)\right|_{\max }$ from (C.7) into (C.5) gives

$$
\left.\varepsilon_{11}\right|_{\max } \approx-0.435 \frac{K_{I I}}{E_{m} \sqrt{d^{*}}} .
$$

Note the minus sign. This implies that $\left.\varepsilon_{11}\right|_{\max }<0$ for $K_{I I}>0$ (positive shear stress for a standard Mode II crack) and thus $\left.\varepsilon_{11}\right|_{\max }>0$ for $K_{I I}<0$. For the present problem (Fig. 4.1) the crack tip is subjected to $K_{I I}<0$. This is seen as follows. Coming from the stress-free end of the broken fibre, it is obvious that the strain in the fibre is less than in the surrounding matrix. Then the displacement of the matrix $u_{m}$ (upper crack face) in the $x_{1}$ direction will be smaller in magnitude than the displacement of the fibre $u_{f}$ (lower crack face), in accordance with $K_{I I}<0$, see Fig. 4.1. 
With $K_{I I}<0$ we obtain by setting $\mathrm{G}=\mathrm{G}^{i}$ in (2.3)

$$
K_{I I}=-\sqrt{\mathrm{G}^{i} \bar{E}_{*}} .
$$

Inserting $K_{\text {II }}$ from (C9) into (C.8) and using Hooke's law (C.3) gives

$$
\hat{\sigma}_{f}^{K}=\left.\sigma_{f}^{K}\right|_{\max }=0.435 \frac{E_{f}}{E_{m}} \sqrt{\frac{\mathrm{G}^{i} \bar{E}_{*}}{d^{*}}} .
$$

\section{Acknowledgements}

This study was partially funded by the "Danish Centre for Composite Structure and materials for Wind Turbines (DCCSM)", fund no. 09-067212 from the Danish Council for Strategic Research. Useful discussions with professor Ramesh Talreja, Dr. Povl Brøndsted and Dr. Hans Lilholt are acknowledged.

\section{References}

Abaqus. Dassault Systemes Dassault System Corp, RI, USA, 2017.

Ahn, B. K., and Curtin, W. A., 1997. Strain and hysteresis by stochastic matrix cracking in ceramic matrix composites, J. Mech. Phys. Solids. 45, 177-99.

Alves, M., and Pimenta, S., 2018. A computationally-efficient micromechanical model for the fatigue life of unidirectional composites under tension-tension loading, International Journal of Fatigue 116, 677-690.

Beyerlein, I. J., and Phoenix, S. L., 1996. Stress concentrations around multiple fiber breaks in an elastic matrix with local yielding or debonding using quadratic influence superposition, J. Mech. Phys. Solids 44, 1997-2039. 
Budiansky, B., Hutchinson, J. W., and Evans, A. G., 1986. Matrix fracture in fiber-reinforced ceramics, J. Mech. Phys. Solids. 34,167-78.

Cho, C., Holmes, J. W., and Barber, J. R., 1991. Estimation of interfacial shear in ceramic composites from frictional heating measurements, J. Am. Ceram. Soc. 74, 2808-8.

Dundurs, J., 1969. Discussion on 'Edge-bonded dissimilar orthogonal elastic wedges under normal and shear loading', J. Appl. Mech. 36, 650-2.

Feih, S. Thraner, A., And Lilholt, H., 2005. Tensile strength and fracture surface characterisation of sized and unsized glass fibers, Journal of Materials Science 40, 1615-23.

Gamstedt, E. K., 2000. Effects of debonding and fiber strength distribution on fatigue-damage propagation in carbon fiber-reinforced epoxy, Journal of Applied Polymer Science 76, 457-74.

Gamstedt, E. K., and Talreja, R., 1999. Fatigue damage mechanisms in unidirectional carbonfibre-reinforced plastics, Journal of Materials Science 34, 2235-46.

Goutianos, S., and Peijs, T., 2001. Fatigue damage mechanisms in carbon-epoxy multi-fibre model composites, Advanced Composites Letters 10, 2,1-32.

Graciani, E., Varna, J., Mantic, V.,Blazquez, A. and Paris, F., 2011. Evaluation of interfacial fracture toughness and friction coefficient in the single fiber fragmentation test. Procedia Engineering 10, 2478-83.

He, M. -Y. and Hutchinson, J. W., 1989. Crack deflection at an interface between dissimilar elastic materials, International Journal of Solids and Structures 25, 1053-67.

Hutchinson, J. W., and Jensen, H. M., 1990. Models of fiber debonding and pullout in brittle composites with friction, Mechanics of Materials 9, 139-63.

Hutchinson, J. W., and Suo, Z., 1992, Mixed mode cracking in layered materials, in Advanced in Applied Mechanics, Vol. 29 (Ed. J. W. Hutchinson and T. Y. Wu), Academic Press Inc., Boston, 63191. 
Jamison, R. D., Schulte, K., Refsnider, K. L., and Stinchcomb, W. W., 1984. Characterization and analysis of damage mechanisms in tension-tension fatigue of graphite/epoxy laminates, Effects of Defects in Composite Materials, ASTM STP 836, American Society for Testing and Materials, 21-55. Jero, P. D., and Kerans, R. J., 1990. The contribution of interfacial roughness to sliding friction of ceramic fibers in a glass matrix, Scripta Metall. Mater. 24, 2315-8.

Kanninen, M. F., and Popelar, C. H., 1985. Advanced fracture mechanics, Oxford University Press, New York, p. 145, p. 500.

Keith, W. P., and Kedward, K. T., 1995. The stress-strain behavior of a porous unidirectional ceramic matrix composite, Composites 26, 163-74.

Kim, B. W., and Nairn, J. A., 2002. Experimental verification of the effect of friction and residual stress on the analysis of interfacial debonding and toughness in single fiber composites. Journal of Materials Science 37, 3965-72.

Mackin, T. J., Warren, P. D. and Evans, A. G., 1992. Effects of fiber roughness on inerface sliding in composites, Acta Metallurgica et Materialia 40, 1251-7.

Mandell, J. F., Samborsky, D. D., Wang, L. and Wahl, N. K., 2003. New fatigue data for wind turbine blade materials, Journal of Solar Energy 125, 506-14.

Marshall, D. B., Shaw,M. C., and Morris, W. L., 1992. Measurement of interfacial debonding and sliding resistance in fiber reinforced intermetallics, Acta Metall. Mater. 40, 443-54.

McMeeking, R. M., and Evans, A. G., 1990. Matrix fatigue cracking in fiber composites, Mechanics of Materials 9, 217-27.

Nijssen, R., 2012. OptiDat Database, Knowledge Centre WMC, http://www.wmc.eu/optimatblades_optidat.php

Paris, P., and Erdogan, F., 1963. A critical analysis of crack propagation laws, Journal of Basic Engineering, Series D 85, 528-34. 
Parmigiani, J. P., and Thouless, M. D., 2007. The effects of cohesive strength and toughness on mixed-mode delamination of beam-like geometries, Engineering Fracture Mechanics 74, 2675-99. Partridge, I., Virlouvet, P., Chubb, J., and Curtis, P., 1989. Effect of fibre volume fraction on tensile fatigue behaviour of UD glass/epoxy composite, in proceeding Developments in the science and technology of composite materials, Third European Conference on Composite Materials,

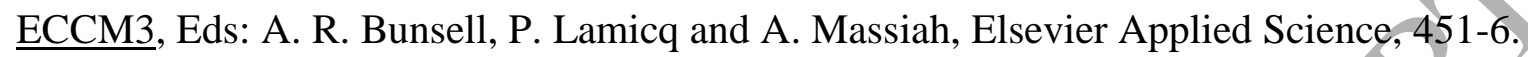

Press, W. H., Teukolsky, S. A., Vetterling, W. T., and Flannery, B. P., 1992. Numerical recipes in Fortran. The art of scientific computering. 2nd edition. Cambridge University Press, p. 355.

Pryce, A. W., and Smith, P. A., 1993. Matrix cracking in unidirectional ceramic matrix composites under quasi-static and cyclic loading, Acta Metall. Mater. 41, 1269-81.

Pupurs, A., and Varna. J., 2013. Energy release rate based fiber/matrix debond growth in fatigue. part I: self-similar crack growth, Mechanics of Advanced Materials and Structures 20, 276287.

Pupurs, A., Goutianos, S., Brøndsted, P. and Varna, J., 2013. Interface debond crack growth in tension-yension cyclic loading of single fibre polymer composites, Composites Part A. 44, 86-94.

Rice, J. R., 1968. A path independent integral and the approximate analysis of strain concentrations by notches and cracks, J. Appl. Mech. 35, 379-86.

Rice, J. R., 1988. Elastic fracture mechanics concepts for interfacial cracks, J. Appl. Mech. 55, 98103.

Rouby, D., and Reynaud, P., 1993. Fatigue behaviour related to the interface modification during load cycling in ceramic-matrix composites, Comp. Sci. Techn. 48, 109-118.

Samborsky, D. D., Agastra, P., and Mandell, J. F., 2008. Effects of glass fabric and laminate construction on the fatigue of resin infused blade materials, Proc. 2008 Wind Energy Symposium, 7-10 January 2008, Reno, Nevada, USA. American Institute of Aeronautics and Astronautics (AIAA). 
Suo, Z., and Hutchinson, J. W., 1990. Interface crack between two elastic layers, Int. J. Fract. 43, 118.

Suo, Z., Bao, G., and Fan, B., 1992. Delamination R-curve phenomena due to damage, J. Mech. Phys. Solids. 40, 1-16.

Sørensen, B. F., 2017. Micromechanical model of the single fiber fragmentation test, Mechanics of Materials 104, 38-48.

Sørensen, B. F., J. W. Holmes, J. W., and Vanswijgenhoven, E. L., 2002, Does a true fatigue limit exist for continuous fiber-reinforced ceramic matrix composites?, Journal of the American Ceramic Society, 85, 359-65.

Talreja, R., 1981. Fatigue of composite materials: Damage mechanisms and fatigue life diagrams, Proc. Royal. Soc. London A378, 461-75.

van den Heuvel, P. W. J., Goutianos, S., Young, R.J., and Peijs, T., 2004. Failure phenomena in multiple-fibre model composites - part 6. A finite element study on the stress concentrations in unidirectional carbon fibre-reinforced epoxy composites, Comp. Sci. Techn. 64, 645-56.

van den Heuvel, P. W. J., Peijs, T., and Young, R. J., 1997. Failure phenomena in multiple-fibre model composites: Part 1. An experimental investigation into the influence of fibre spacing and fibre-matrix adhesion, Composites part A 27, 855-9.

van den Heuvel, P. W. J., Peijs, T., and Young, R. J., 1998. Failure phenomena in multiple-fibre model composites - 3. A Raman spectroscopic study on the influence of interfacial debonding on the stress concentrations, Composite Science and Technology 58, 933-44.

Weibuli, W., 1939. A statistical theory of the strength of materials, Ingeniörsvetenskapsakademiens Handlingar, 151, Generalstabens Litografiska Anstalts Förlag, Stockholm.

Wiederhorn, S. M., 1967. Influence of water vapor on crack propagation in soda-lime glass, J. Am. Ceram. Soc. 50, 407-14. 
Zangenberg, J., Brøndsted, P., and Gillespie, J. W., 2013. Fatigue damage propagation in unidirectional glass fibre reinforced composites made of non-crimp fabrics, Journal of Composite Materials 48, 2711-27.

Zhou, Y., and Mallick, P. K., 2004. Fatigue strength characterisation of E-glass fibers using fiber bundle test, Journal of Composite Materials 38, 2025-35.

Zike, S., Sørensen, B. F. and Mikkelsen, L. P., 2016. Experimental determination of the micro-scale strength and stress-strain relation of an epoxy resin, Materials \& Design 98, 47-60.

\section{List of symbols}

$A_{1} \quad$ utility parameter (friction and R-ratio)

$E_{f} \quad$ Young's modulus of fibre

$E_{m} \quad$ Young's modulus of matrix

$\bar{E}_{*} \quad$ bimaterial Young's modulus

$\mathrm{G}_{c}^{i} \quad$ interfacial fracture energy

$\mathrm{G}_{\text {min }}^{i}$ energy release rate at minimum applied stress

$K_{I I}^{\max }$ maximum value of Mode II stress intensity factor

$K_{I I}^{\min }$ minimum value of Mode II stress intensity factor

$L_{0} \quad$ reference length (Weibull model)

$P_{f} \quad$ probability of failure

$R \quad$ R-ratio $\left(R=\bar{\sigma}_{\text {min }} / \bar{\sigma}_{\text {max }}\right)$

$R_{m} \quad$ outer radius of matrix cylinder 


\section{$S \quad$ surface area}

$S_{F} \quad$ surface area with frictional sliding

$V_{f} \quad$ fibre volume fraction

$d^{*} \quad$ fibre spacing

$g(\theta)$ angular function

$\ell_{d} \quad$ debond length (first loading)

$\ell_{d}^{c} \quad$ debond length (cyclic loading)

$\ell_{s}^{l c} \quad$ forward slip length during cyclic reloading

$\ell_{s s}^{l c} \quad$ maximum length of forward slip length (cyclic loading)

$\ell_{s}^{u c} \quad$ reverse slip length (cyclic unloading)

$\ell_{s s}^{u c} \quad$ maximum length of reverse slip length (cyclic loading)

$m \quad$ Weibull modulus

$r \quad$ fibre radius

$r^{*} \quad$ radial distance (cylindrical coordinate)

$u_{m} \quad$ displacement of the matrix

z coordinate axis

$\Delta \varepsilon_{c}$ strain change in non-slip zones

$\Delta \varepsilon^{T}$ mismatch strain

$\Delta u \quad$ displacement difference (slip) between fibre at matrix

$\Delta K_{I I}$ Mode II stress intensity factor range 
$\left(\Delta K_{I I}\right)_{t h}$ threshold value of stress intensity factor range

$\Pi$ potential energy

$\varepsilon_{11}$ normal strain component in the matrix

$\bar{\varepsilon}_{f l} \quad$ fatigue limit in terms of strain

$\bar{\varepsilon}_{\max }$ maximum applied strain

$\kappa \quad$ Kolosov constant

$\sigma_{I} \quad$ stresses for State I

$\sigma_{\text {II }} \quad$ stresses for State II

$\sigma_{0} \quad$ characteristic strength (Weibull model)

$\sigma_{f}^{-}$normal stress in fibre (debonded zone)

$\sigma_{f}^{+} \quad$ normal stress in fibre (bonded zone)

$\hat{\sigma}_{f}^{K} \quad$ stress in fibre induced by K-field

$\sigma_{f}^{r e s}$ residual stress in fibre

$\sigma_{f}^{*} \quad$ stress in fibre surface in fibre next to broken fibre

$\sigma_{m}^{-} \quad$ normal stress in matrix (debonded zone)

$\sigma_{m}^{+}$normal stress in matrix (bonded zone)

$\sigma_{m}^{r e s}$ residual stress in matrix

$\bar{\sigma} \quad$ applied stress

$\bar{\sigma}_{f l} \quad$ fatigue limit in terms of stress

$\bar{\sigma}_{i} \quad$ applied stress at which the debond crack growth can initiate

$\bar{\sigma}_{\max }$ maximum applied stress 


\section{$\bar{\sigma}_{\min }$ minimum applied stress}

$\varphi \quad$ angle around the circumference of fibre

$\tau_{s} \quad$ interfacial sliding shear stress (first loading)

$\tau_{s}^{u} \quad$ interfacial sliding shear stress (first unloading)

$\tau_{s}^{c} \quad$ interfacial sliding shear stress after many cyclic forward/reverse slips

$\theta \quad$ angle (cylindrical coordinate)

$\xi_{F} \quad$ frictional energy dissipation 